\title{
Non-Covalent Organocatalyzed Domino Reactions Involving Oxindoles: Recent Advances
}

\author{
Tecla Gasperi $^{1, *}$ (D), Martina Miceli ${ }^{1}{ }^{(\mathbb{D})}$, Jean-Marc Campagne ${ }^{2}$ \\ and Renata Marcia de Figueiredo $2, *$ \\ 1 Dipartimento di Scienze, Sezione di Nanoscienze e Nanotecnologie, Università degli Studi Roma Tre, \\ via della Vasca Navale 79, I-00146 Roma, Italy; martina.miceli@icloud.com \\ 2 ICGM-Institut Charles Gerhardt Montpellier, UMR 5253 CNRS-UM-ENSCM, Ecole Nationale Supérieure \\ de Chimie, 8, Rue de l'Ecole Normale, 34296 Montpellier CEDEX 5, France; jean-marc.campagne@enscm.fr \\ * Correspondence: tecla.gasperi@uniroma3.it (T.G.); renata.marcia_de_figueiredo@enscm.fr (R.M.d.F.); \\ Tel.: +39-06-5733-3371 (T.G.); +33-467-147-224 (R.M.d.F.)
}

Received: 16 September 2017; Accepted: 26 September 2017; Published: 29 September 2017

\begin{abstract}
The ubiquitous presence of spirooxindole architectures with several functionalities and stereogenic centers in bioactive molecules has been appealing for the development of novel methodologies seeking their preparation in high yields and selectivities. Expansion and refinement in the field of asymmetric organocatalysis have made possible the development of straightforward strategies that address these two requisites. In this review, we illustrate the current state-of-the-art in the field of spirooxindole synthesis through the use of non-covalent organocatalysis. We aim to provide a concise overview of very recent methods that allow to the isolation of unique, densely and diversified spirocyclic oxindole derivatives with high structural diversity via the use of cascade, tandem and domino processes.
\end{abstract}

Keywords: spirooxindole derivatives; non-covalent organocatalysis; hydrogen-bonding; cascade; tandem; domino; enantioselectivity; chiral building blocks

\section{Introduction}

Heterocyclic compounds are found in a broad range of bioactive molecules, natural products, and drugs. Consequently, several novel efficient strategies based on catalytic methods have been validated to date for the direct assessment of such scaffolds in an enantiopure fashion [1-3]. In this context, spirocyclic oxindole derivatives have appeared as privileged structural motifs being part of a great number of synthetic and natural products displaying remarkable biological activities as well as useful biomedical applications (Figure 1) [4-6]. Asymmetric organocatalysis has appeared as an appealing tool in order to prepare such compounds with rich structural diversity and complexity through cascade, tandem, and domino processes [7-9]. Indeed, since the beginning of the 21st century, the golden-age of organocatalysis, several reports and substantial advances on the field of the synthesis of complex structural entities via the combination of organocatalysis and cascade transformations have been reported so far. The ability to reach for molecular complexity through the conscious choice of substrates and catalysts in a single transformation has inspired a growing number of research groups. Seminal reports that cover this subject have appeared before 2015 [10-13].

The aim of this review is to describe recent advances towards the stereocontrolled synthesis of strained spiro-quaternary stereocenters on the oxindole core through non-covalent organocatalysis [14-16] within the timeframe from 2015 to the middle of 2017. It is the authors' aim to attract the reader's attention to the potential of asymmetric non-covalent organocatalysis to mediate one-pot cascade, tandem, and domino processes that, employing an oxindole derivative as starting material, give a facile access to complex molecules featured by several functionalities and various stereogenic centers. 


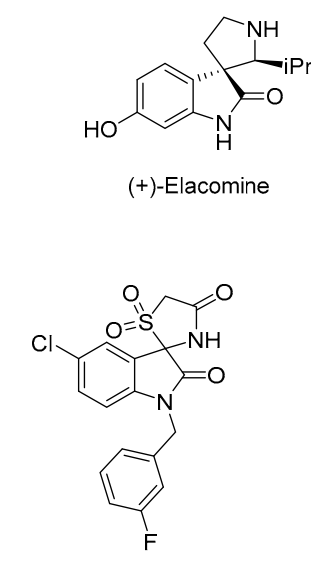

Anti-tubercolosis agent

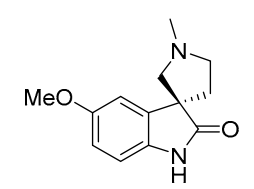

$(-)$-Horsfiline
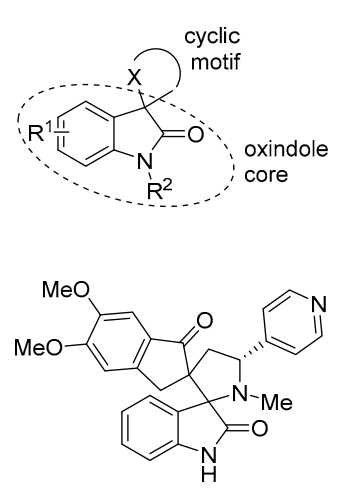

AChE Inhibitor

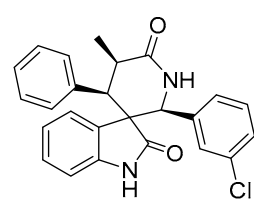

MDM2 Inhibitor

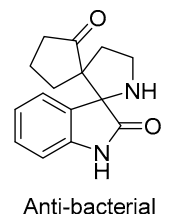

Figure 1. Examples of bioactive compounds built around the oxindole framework.

The observed high yields and remarkable stereoinduction, which resemble Nature's outcomes, mainly rely on H-bond interactions, electrostatic effects, and $\pi-\pi$ staking that are established between the oxindole core, the employed reagent, and the selected catalyst (i.e., dual and cooperative catalysis) [17]. Examples of such connections are depicted in Figure 2.

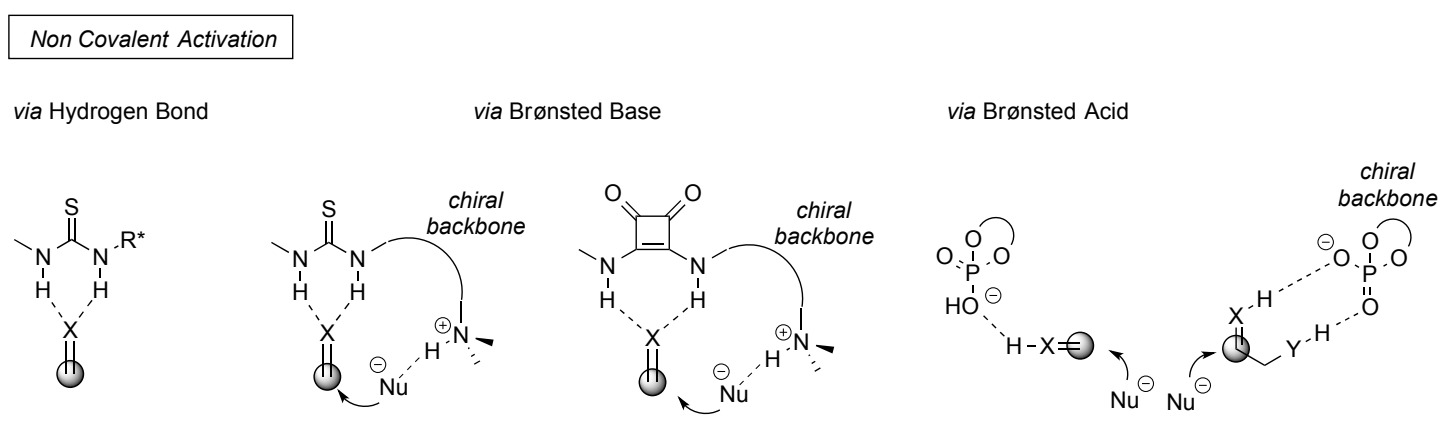

Examples with oxindole derivatives

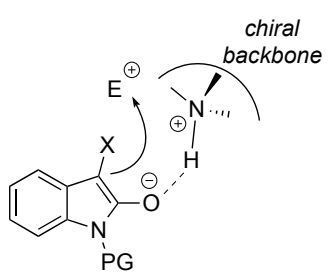

$P G$

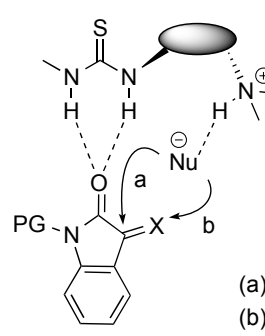

(a) mostly when $\mathrm{X}=\mathrm{O}, \mathrm{NR}^{1}$ (b) mostly when $X=C R^{1} R^{2}$

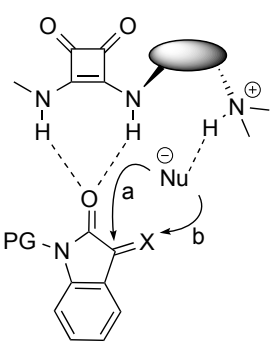

Figure 2. Examples of non-covalent interactions in a substrate/reagent/catalyst system.

Far to be exhaustive and comprehensive, the current review is divided according to the most employed class of catalysts. Specifically, after a quick overview about the Cinchona alkaloids (Section 1), the subsequent synthetic elaborations of these natural-occurring organocatalysts are going to be introduced (Sections 2-5); meanwhile, Section 6 will detail an example of non-covalent activation via Brønsted acids. Finally, we apologize for any omissions. 


\section{Cinchona Alkaloid Catalysts}

Discovered in 1820 for its antimalarial properties, Cinchona alkaloids have found several applications in various and quite different fields ranging from medicine to food and beverage industries, and even including organic chemistry. Besides the initial uses as resolving agents, over the last decades, Cinchona alkaloids have established a primary and outstanding role in asymmetric synthesis as highly efficient organocatalysts capable of promoting a wide range of enantioselective transformations in both homogeneous and heterogeneous environments [18]. The impressive chiral induction mainly relies both on the accessibility of different chiral skeletons and on the facile adaptability to various reaction processes (Figure 3). Specifically, the 1,2-aminoalcohol group summarizes in one molecule:

(i) the basicity and bulkiness of quinuclidine moiety, apt to activate a nucleophile by deprotonation as well as to stabilize the developing positive charge,

(ii) the secondary 9-hydroxy group, which acts as both an acid and a H-bond donor, and is suitable for further chemical modification of the catalyst structure. Additionally, the overall catalytic action is also ascribed to the possible $\pi-\pi$ interactions with the aromatic quinoline ring.

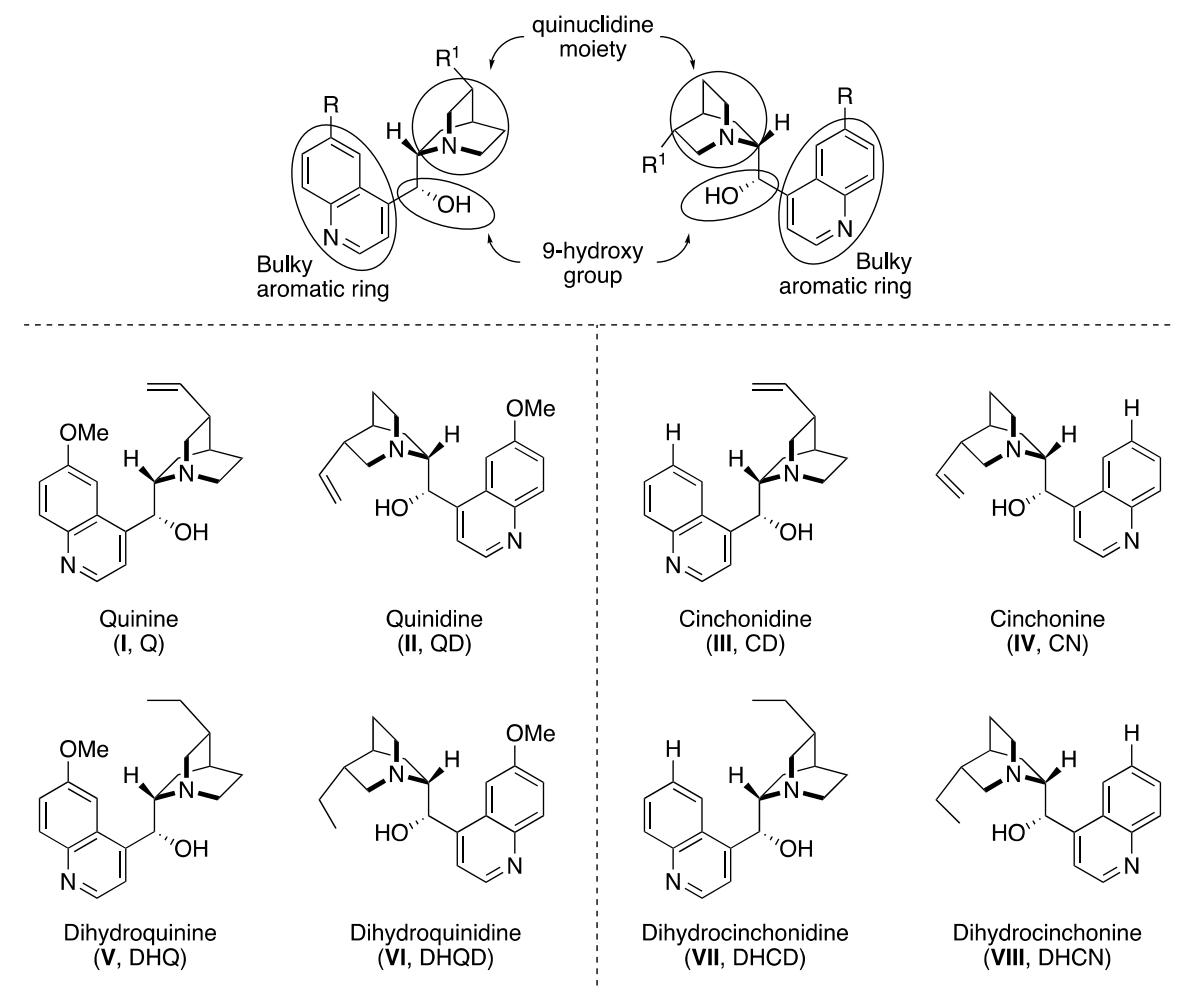

Figure 3. Representative examples of Cinchona alkaloids.

Representative examples of widespread used Cinchona alkaloids are depicted in Figure 3. Notably, as fragments of more complex organocatalysts (Sections 3-5) the same structures play a crucial role to induce high levels of diastereo- and enantioselectivity.

Within this context, in 2015, Yuan and co-workers succeeded in the construction of a class of spirocyclic oxindoles through a domino Mannich-cyclization process [19]. Specifically, employing various 3-isothiocyanate oxindoles $\mathbf{1}$ and imines $\mathbf{2}$ as substrates, the simplest commercially available quinine (I, $1 \mathrm{~mol} \%$ ) made possible the diastereo- (up to $>99: 1$ d.r.) and enantioselective (up to $97 \%$ ee) synthesis of spiro[imidazolidine-2-thione-4,3'-oxindole] derivatives 3 (Scheme 1). Notably, the optimized conditions, i.e., using toluene as solvent at $0{ }^{\circ} \mathrm{C}$ with the addition of $4 \AA$ molecular sieves (MS), led, in just $10 \mathrm{~min}$, to the desired products isolated in remarkable yields (up to $95 \%$ ). 

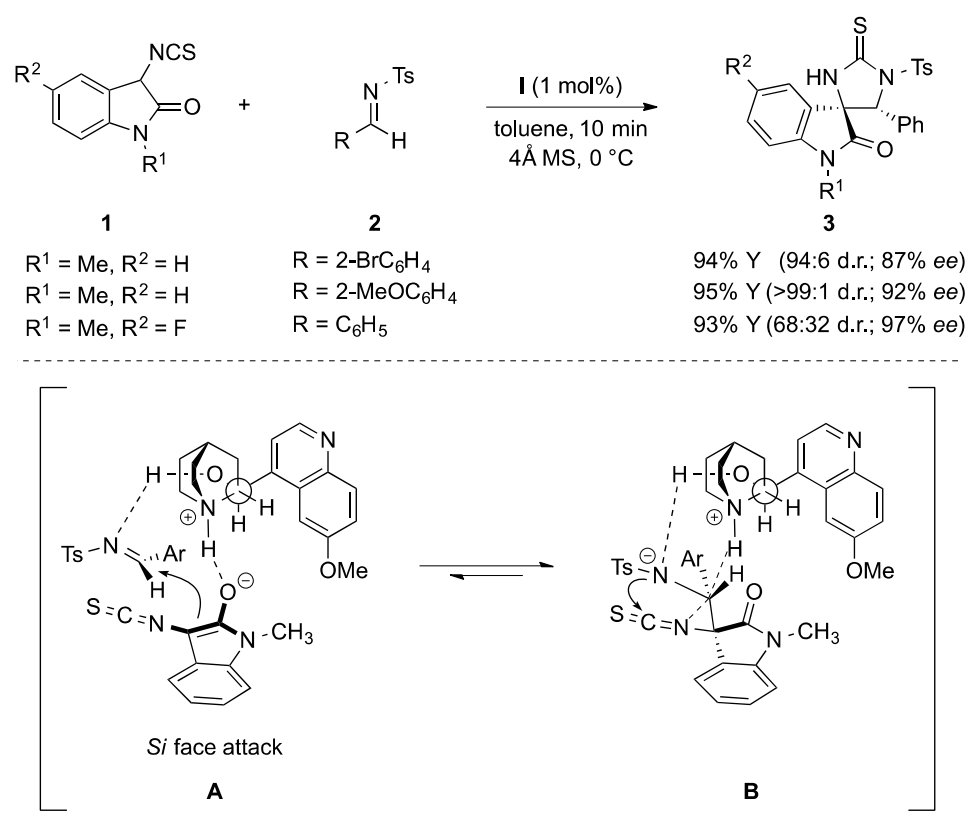

Scheme 1. Synthesis of spiro[imidazolidine-2-thione-4, $3^{\prime}$-oxindole] via domino Mannich/Cyclization process and proposed transition states where $\mathbf{A}$ and $\mathbf{B}$ are the suggested intermediates $(\mathrm{Y}=$ Yield).

To explain the observed outstanding stereocontrol, the authors suggest that in the transition state the quinine simultaneously activates: (i) the tosyl-protected imine via a H-bond interaction of the $\mathrm{C} 9-\mathrm{OH}$ and (ii) the 3-isothiocyanate oxindole moiety via deprotonation and consequent enolization performed by the tertiary amine group. In such a way, the imine $S i$-face is exposed to the attack of the Si-face nucleophile counterpart (A, Scheme 1). The subsequent ring closure reaction involves the just formed $N$-nucleophile and the electron-poor carbon of the isocyanate oxindole framework (B, Scheme 1).

More recently the impressive stereoinduction properties of Cinchona alkaloids have been validated even in a kinetic resolution approach. Indeed, Tanaka and co-workers were engaged in the synthesis of spirooxindole polycycles 8 bearing a spiro[4,5]decane system that was remarkably accomplished by a two-step strategy involving a formal [4 +1] cycloaddition and a subsequent Michael-Henry cascade transformation (Scheme 2) [20].

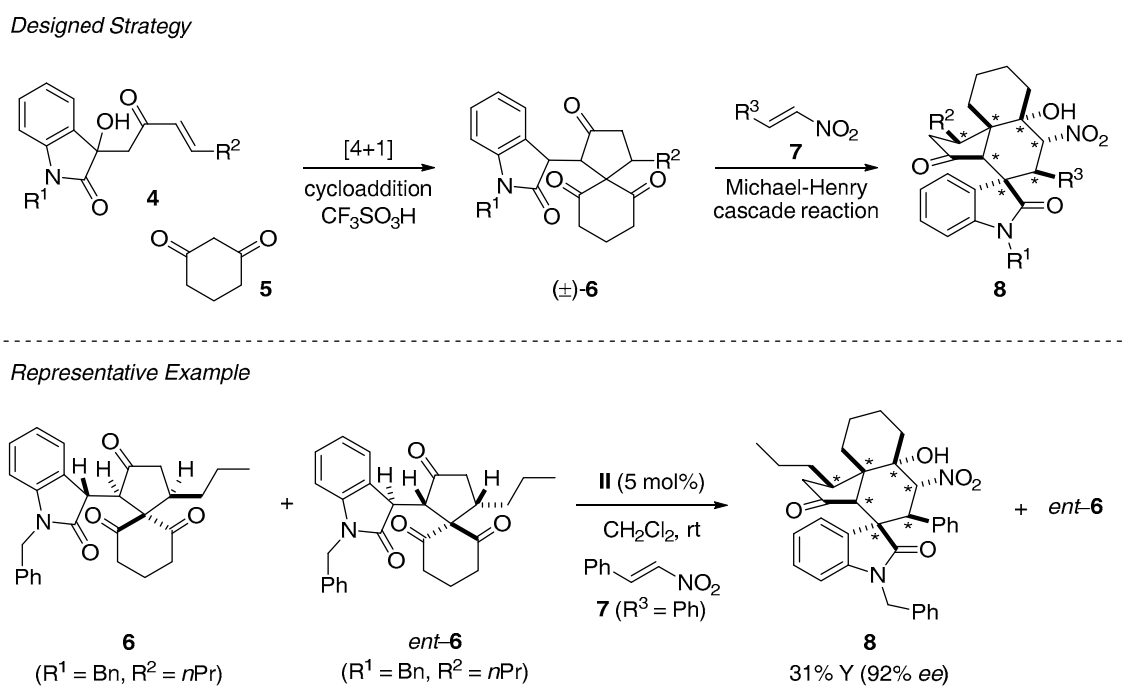

Scheme 2. Synthesis of spirooxindole polycycles bearing a spiro[4,5]decane system. 
While the former reaction was carried under acidic catalysis, the Michael-Henry cascade steps were performed under quinidine (II) catalysis at room temperature. In such conditions, the final polycyclic derivatives 8 featuring seven stereogenic centers were isolated in high optical purity (80-92\% ee) despite modest yields (up to $28 \%$ ) consequence of the racemic mixture used as starting materials. However, the unreacted enantiomer ent-6 was easily recovered in enantioenriched fashion (up to $43 \%$ yield, $92-98 \%$ ee).

Although initially the simplest naturally available Cinchona alkaloids have been widely exploited, over the years, organic chemists have created more efficient and complex molecules bearing the same scaffold that was implemented with the introduction of further functionality capable of $\mathrm{H}$-bond interactions. In the following sections, we are going to illustrate and compare other privileged organic chirality inducers, most of which could be added to the realm of Cinchona alkaloid derivatives.

\section{3. (DHQD) $)_{2}$ Based Catalysts}

Joining together two units of Cinchona derivatives/analogues in a single molecule, creating the so-called bis-Cinchona alkaloids, is the easiest way to enhance the H-bond network inside the catalyst/substrates system. Such structures have mainly shown their efficiency as chiral ligands in the Sharpless dihydroxylation. However, over the years, several research groups have highlighted their potential and effectiveness as simple organocatalysts without the introduction of further metal salt additives. Among others, $\mathrm{Wu}$ and co-workers exploited the ability of (DHQD) ${ }_{2}$ PYR (IX) in Michael/cyclization cascade reaction to synthesize different spirocyclic oxindoles starting from isatilidene malonitriles as initial electrophile. Precisely, the employment of acyclic $\beta, \gamma$-unsaturated amides $\mathbf{1 0}$ as vinylogous enolates, smoothly provided the titled spirooxindoles $\mathbf{1 1}$ in good yields $(87-95 \%)$ and noteworthy enantioselectivity $(77-96 \%$ ee) even though the overall outcomes were affected by the steric hindrance introduced on both donor and acceptor reagents (Scheme 3) [21].

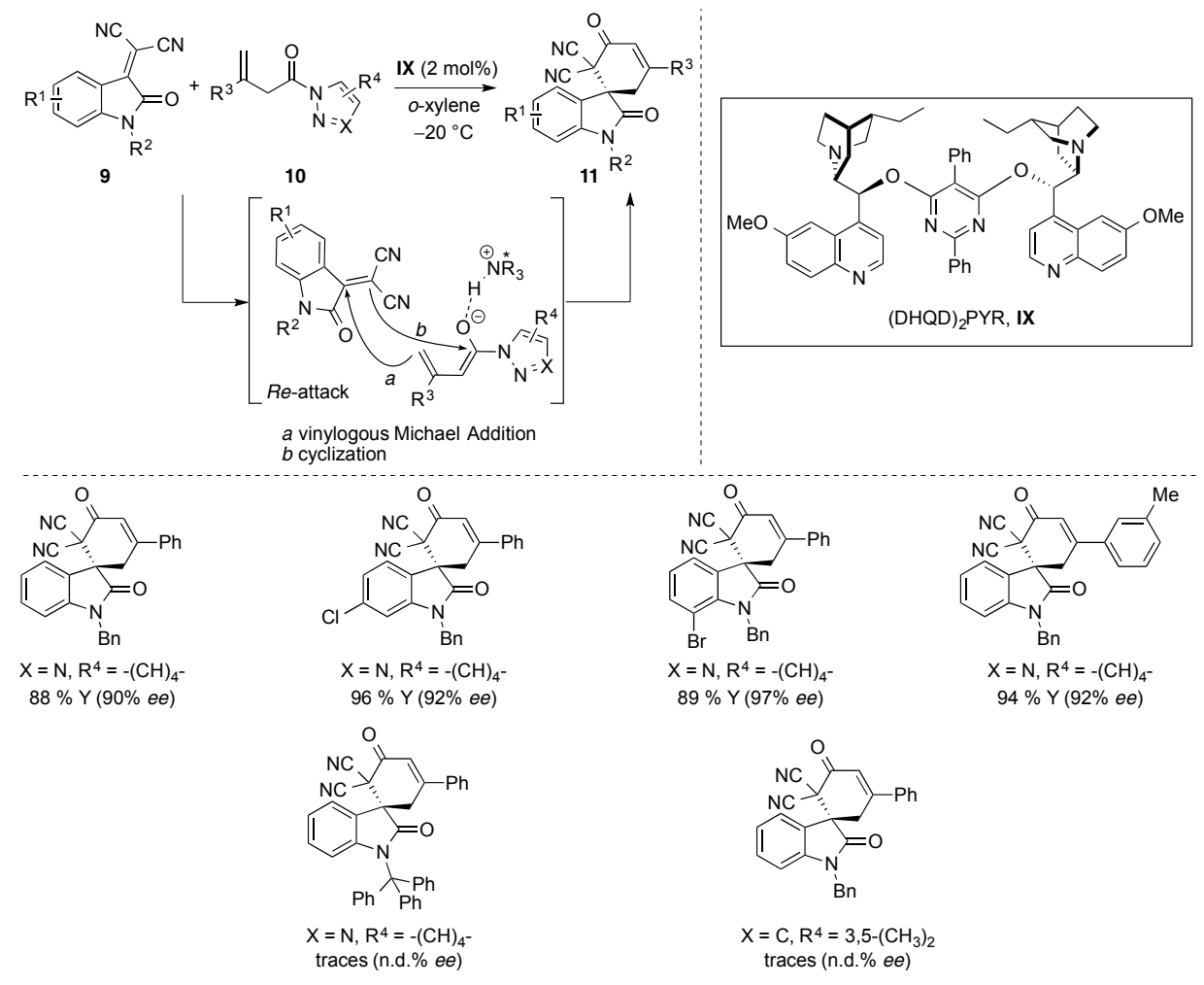

Scheme 3. Michael/cyclization cascade reaction to spirociclyc oxindoles catalyzed by (DHQD) ${ }_{2} \mathrm{PYR}$ (IX).

Likewise, the replacement of the Michael donor with suitable pyrazolone 12 furnished the expected spiro[indoline-3, $4^{\prime}$-pyrano[2,3-c]pyrazole] derivatives $\mathbf{1 3}$ by performing the reaction in 
analogous conditions (i.e., $-20{ }^{\circ} \mathrm{C}$, AcOEt as solvent) and adding only $1 \mathrm{~mol} \%$ of the chosen organocatalyst IX (Scheme 4) [22]. The whole process was completed in a shorter reaction time (from $10 \mathrm{~min}$ to $9 \mathrm{~h}$ ) with respect the previous vinylogous Michael/cyclization sequence (from $60 \mathrm{~min}$ to 7 days) and provided the products in notable yields (96-99\%) and good-to-moderate optical purity $(47-91 \% e e)$.

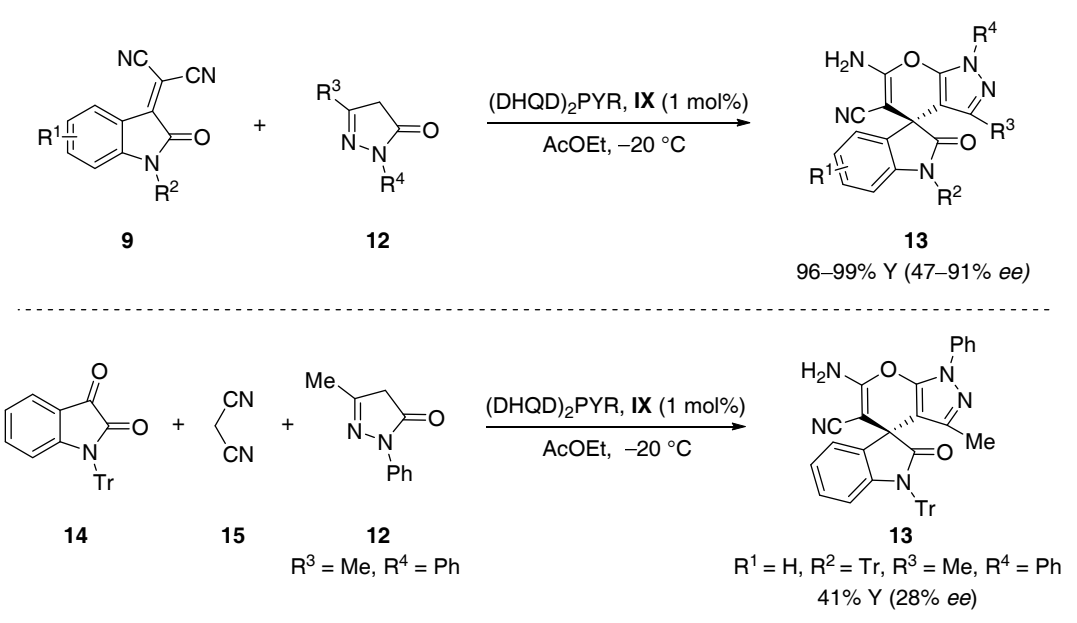

Scheme 4. Michael/cyclization cascade reaction catalysed by (DHQD) ${ }_{2} \mathrm{PYR}$ (IX) when pyrazolone 12 was employed as Michael donor.

Unfortunately, the optimized protocol failed when the one-pot three component reaction of $N$-trityl isatin (14), malonitrile (15), and pyrazolone $12\left(\mathrm{R}^{3}=\mathrm{Me}, \mathrm{R}^{4}=\mathrm{Ph}\right)$ was attempted affording the hypothesized spirocompound $\mathbf{1 3}\left(\mathrm{R}^{1}=\mathrm{H}, \mathrm{R}^{2}=\mathrm{Tr}, \mathrm{R}^{3}=\mathrm{Me}, \mathrm{R}^{4}=\mathrm{Ph}\right)$ in low yield $(41 \%)$ and poor enantioselectivity $(28 \% e e)$.

Almost simultaneously, Enders and co-workers designed and realized an organocatalytic Mannich/Boc-deprotection/aza-Michael sequence of $N$-Boc ketimine 16 and 3-substituted oxindoles 17 that straightforwardly afforded the functionalized 3,3'-pyrrolidinyl derivatives 18 bearing three stereocenters, two of which were contiguous spiro-stereocenters [23]. Therefore, the devised and validated protocol (i.e., room temperature, MTBE as solvent), which relied on the efficiency of (DHQD) ${ }_{2}$ PHAL $\mathbf{X}(10 \mathrm{~mol} \%)$ as catalyst, gave access in moderate-to-good yields (41-84\%) and high stereoselectivity (up to >20:1 d.r., 90-98\% ee) to complex spirocyclic systems 18 (Scheme 5).<smiles>[R1]N1C(=O)C(=N[R16](C)(C)C)c2cc[R1]cc21</smiles>

16

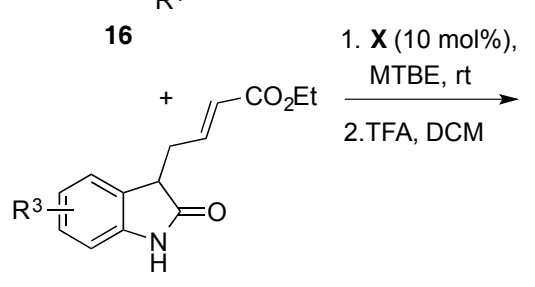
17

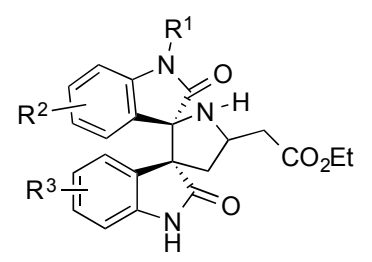

18

$41-84 \% Y$

(up to $>20: 1$ d.r.; $90-98 \%$ ee)

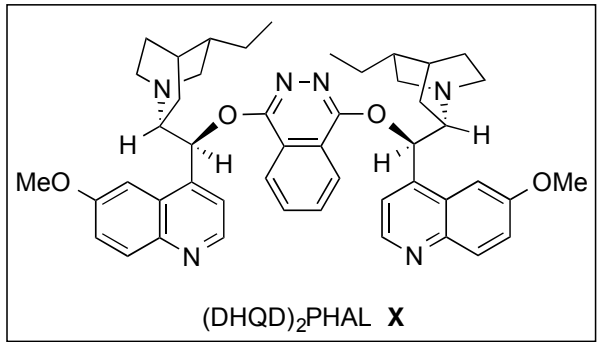

$(\mathrm{DHQD})_{2}$ PHAL $\mathbf{X}$

Scheme 5. Organocatalytic Mannich/Boc-deprotection/aza-Michael sequence developed by Enders and co-workers. 


\section{Thiourea-Based Catalysts}

Thiourea-based organocatalysts [24-27] have been intensively considered for promoting multiple $\mathrm{C}-\mathrm{C}$ and $\mathrm{C}$-heteroatom bonds formation via domino reactions through H-bond network between substrates and catalysts. In this section, recent selected cascade reactions involving such organocatalysts (Figure 4) allowing to highly functionalized chiral spirooxindole-bearing compounds are going to be discussed.<smiles>CN1CCC[C@H]1[C@H](NC(=S)Nc1cc(C(F)(F)F)cc(C(F)(F)F)c1)c1ccccc1</smiles>

$\mathbf{X I}$<smiles>C=CC1C2CCN1C(C(NC(=S)NC(c1ccccc1)[C@H](NC)c1ccccc1)c1ccnc3ccc(OC)cc13)C2</smiles><smiles>C=CC1=CN=C([C@H](NC(=S)NC(c2ccccc2)c2ccccc2)c2ccnc3ccc(OC)cc23)CC1</smiles>

XVII<smiles>C=CC1=CNC([C@H](NC(=S)Nc2cc(C(F)(F)F)cc(C(F)(F)F)c2)c2ccnc3ccc(OC)cc23)CC1</smiles>

$\mathrm{xx}$<smiles>CN(C)C(c1ccccc1)C(NC(=S)Nc1cc(C(F)(F)F)cc(C(F)(F)F)c1)c1ccccc1</smiles>

XII<smiles>CCC1CC2CCN1CC2NC(=S)Nc1ccc(C(F)(F)C(F)(F)F)cc1</smiles>

XV<smiles>CC(NC(=S)Nc1cc(C(F)(F)F)cc(C(F)(F)F)c1)O[C@H](CN1CCCC1)C(C)O[Sb]</smiles>

XVIII<smiles>CC(C)(C)[C@H](CN1CCCCC1)NC(=S)Nc1cc(C(F)(F)F)cc(C(F)(F)F)c1</smiles><smiles>CN(C)C1CCCCC1NC(=S)Nc1cc(C(F)(F)F)cc(C(F)(F)F)c1</smiles>

XIII<smiles></smiles>

XVI<smiles>C=CC1CC2CCC1N2C(NC(=S)Nc1cc(C(F)(F)F)cc(C(F)(F)F)c1)c1ccnc2ccc(OC)cc12</smiles>

XIX

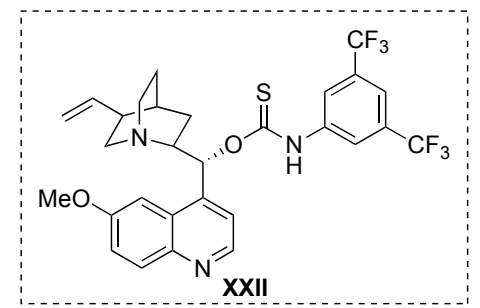

Figure 4. Thiourea and thiocarbamate organocatalysts used in this section.

\subsection{Michael Addition/Cyclization Sequence}

Electrophilic isatilydene malonitrile derivatives 9 are substrates of choice to promote Michael addition/cyclization transformations through reaction with nucleophiles. In 2015, Kesavan and co-workers have indeed devised a sequential vinylogous Michael addition/cyclization in the presence of vinyl malononitriles 19 and $\mathbf{2 0}$ as vinylogous nucleophiles [28]. The reaction, conducted in the presence of L-proline derived bifunctional thiourea catalyst XI $(10 \mathrm{~mol} \%)$ in toluene at $0{ }^{\circ} \mathrm{C}$, demonstrated a wide scope with both substrates (Scheme 6, compounds 21 and 22). In addition, an enantioselective three-component reaction could also be proposed via in situ formation of isatylidene malonitrile derivatives. The authors have also highlighted the ability of XI to afford high levels of enantioselectivity without the need for $\mathrm{N}$-protected oxindoles. Indeed, enantioselective transformations involving oxindoles usually require the prior $\mathrm{N}$-protection in order to avoid unsought substrate interactions with the catalyst. 


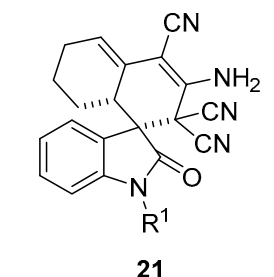

21

$\mathrm{R}^{1}=\mathrm{Me} ; 86 \% \mathrm{Y}(96 \%$ ee $)$ $R^{1}=H ; \quad 88 \% Y\left(99 \% e e^{a}\right)$

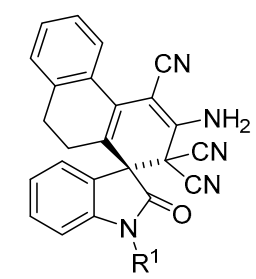

22

$\mathrm{R}^{1}=\mathrm{Me} ; 92 \% \mathrm{Y}(96 \%$ ee $)$

$\mathrm{R}^{1}=\mathrm{H} ; \quad 88 \% \mathrm{Y}\left(99 \% e e^{\mathrm{b}}\right)$
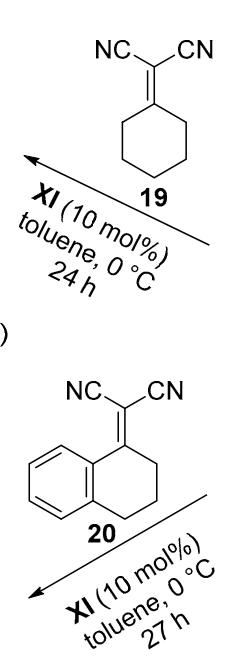<smiles>O=C1CSC(c2ccccc2)=N1</smiles>

27
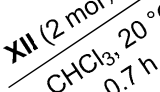

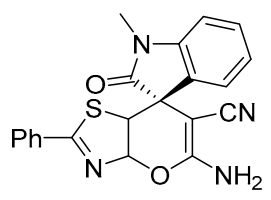

28

$74 \%$ Y $(94 \%$ ee $)$<smiles>[H][R]1C(=O)C(=C(C#N)C#N)c2ccccc21</smiles>

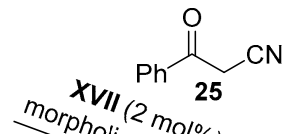

$\mathrm{N}$

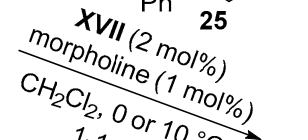<smiles>N#Cc1ccccc1C(=O)Nc1ccccc1</smiles>

26

$\mathrm{R}^{1}=\mathrm{Me} ; 99 \% \mathrm{Y}(88 \%$ ee $)$ $\mathrm{R}^{1}=\mathrm{H} ; \quad 99 \% \mathrm{Y}(76 \%$ ee $)$<smiles>CCOC(=O)C1=C(C(=O)N(Cc2ccccc2)c2ccc(Br)cc2)C2(C(C)=C(N)N(c3ccc(OC)cc3OC)C(N)=C2C#N)C(C(=O)OCC)=C1C</smiles>

24

$82 \%$ Y $(48 \%$ ee $)$

Scheme 6. Recent examples of spirooxindoles synthesized from isatylidene malonitrile derivatives 9 through cascade processes involving Michael addition/cyclization sequence. ${ }^{\text {a }}$ Three-component reaction performed during $36 \mathrm{~h} .{ }^{\mathrm{b}}$ Three-component reaction performed during $48 \mathrm{~h}$.

Herrera and co-workers reported the combination of enamines $\mathbf{2 3}$ and isatylidene malonitrile $\mathbf{9}$ to propose a promising asymmetric synthesis of few 2-oxospiro-[indole-3, $4^{\prime}$-(1 $1^{\prime}, 4^{\prime}$-dihydropyridine)] 24 in moderate yields and enantioselectivities (Scheme 6, compound 24) [29]. The domino process is believed to follow a mechanism that involves a Michael addition, an intramolecular cyclization and then, a tautomerization and is catalyzed by Takemoto's catalyst [30] XIII (30 mol \%) in acetonitrile at $15^{\circ} \mathrm{C}$.

The synthesis of several spiro[4H-pyran-oxindole] derivatives $\mathbf{2 6}$ was proposed by $\mathrm{Wu}$ and co-workers by using $\alpha$-cyano ketones 25 as nucleophiles (Scheme 6) [31]. In the presence of very low loadings of quinidine-derived thiourea organocatalyst XVII (2 mol \%) and morpholine (1 mol \%) in dichloromethane at 0 or $-10{ }^{\circ} \mathrm{C}$, the cascade process takes place within less than two hours and accommodates a broad range of substrates affording the expected products in excellent yields and high enantioselectivities. The authors have shown that the chiral tertiary amine moiety in catalyst XVII is crucial to afford enantioenriched spiro compounds $\mathbf{2 6}$ as in its absence, the expected products were obtained in a racemic manner.

Novel thiazole-fuzed spirooxindoles $\mathbf{2 8}$ were synthesized in high yields and enantioselectivities by using (1R,2R)-1,2-diphenylethane-1,2-diamine derived thiourea catalyst XII (2 mol \%) (Scheme 6) [32]. In this case, the domino transformation also takes place in shorter reaction times and the catalytic system proved to be suitable to a series of 2-substituted thiazol-4-ones 27 as nucleophiles and 2-(1-methyl-2-oxoindolin-3-ylidene)malonitrile as electrophile. Concerning the mechanism, the authors reasoned that the observed stereochemistry of this domino reaction can be explained via a first Michael addition of thiazolones $\mathbf{2 7}$ to 9 to afford the Michael addition intermediate followed by its 
subsequent intramolecular Thorpe-Ziegler-type cyclization. Both steps operate through dual activation of substrates in the presence of the bifunctional thiourea catalyst XII.

The combination of naphthoquinone and chromenone derivatives with oxindole ketoesters as Michael acceptors has paved the way to the preparation of versatile heterocyclic compounds. The group of Kesavan has, once again, underscored proline-based catalyst XI (5 mol \%) as powerful catalyst to carry on tandem Michael addition/hemiketalization of ketoester 29 with 2-hydroxy-1,4-naphthoquinone 30 in dichloromethane at room temperature (Scheme 7) [33]. The expected hybrid spirooxindole-naphthoquinone compounds 31 were obtained in excellent yields and enantioselectivities and displayed good functional tolerance concerning the oxindole ketoester scaffold including different $\mathrm{N}$-protecting groups. However, unprotected (i.e., $\mathrm{N}$-H) oxindole substrates gave lower yields and enantioselectivities probably due to the additional $\mathrm{H}$-bond binding site that might be in competition either with the organocatalyst or with the substrates. Independently and shortly after, the group of Wang proposed a similar transformation in the presence of catalyst $\mathbf{X X}$ (10 mol \%). In this work, a broader scope has been proposed with respect of both substrates [34]. Additionally, the synthesis of several optically active spiro[oxindole-benzo[ $g$ ]chromene-dione] derivates was also described via cascade reaction between 2-hydroxy-1,4-naphthoquinone as nucleophile and oxindole ketoesters.

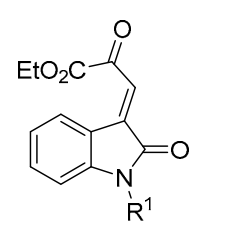

29

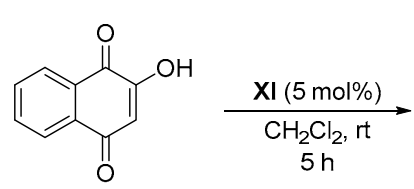

30

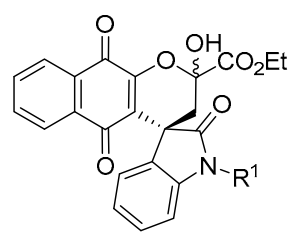

31

$\mathrm{R}^{1}=\mathrm{Me} ; 84 \% \mathrm{Y}(98 \%$ ee $)$

$R^{1}=H ; \quad 78 \% Y\left(60 \% e e^{a}\right)$

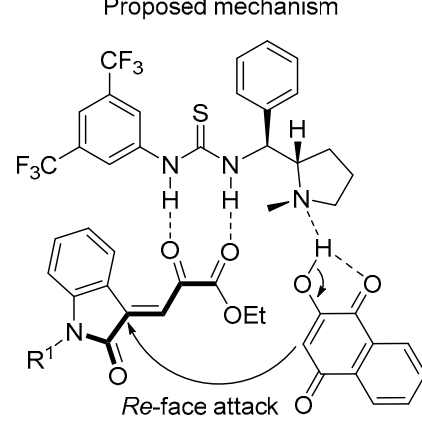

Scheme 7. Synthesis of hybrid spirooxindole-naphthoquinones through catalysis with proline-based catalyst XI. ${ }^{a}$ Reaction was carried out at $5{ }^{\circ} \mathrm{C}$.

In 2016, Enders and co-workers developed a highly selective domino oxa-Michael/1,6-addition sequence to synthesize functionalized chromans with an oxindole moiety [35]. The success of the transformation relies on the use of unprecedented ortho-hydroxyphenyl-substituted para-quinone methide 33 as donor-Michael acceptor substrates in combination with isatin-derived enoate 32 (Scheme 8). The mild reaction conditions [i.e., catalyst XVIII ( $5 \mathrm{~mol} \%$ ) in toluene at room temperature] showed significantly wide substrate scope and functional group tolerance.

Other interesting substrates used in cascade transformations to afford highly substituted and poly-functionalized spirooxindoles through Michael addition/cyclization process are 3-isothiocyanate oxindoles (Scheme 9, compound 35). Indeed, their ambiphilic character (e.g., bearing both electrophilic and nucleophilic sites) allows for the synthesis of several functionalized spirooxindoles via reaction with diversified suitable substrates such as electron-poor olefins. Chowdhury, Ghosh, and co-workers proposed the use of quinine-derived thiourea catalyst XIX $(20 \mathrm{~mol} \%)$ in toluene at $0{ }^{\circ} \mathrm{C}$ in order 
to synthesize a broad range of 3,2'-pyrrolidinyl spirooxindoles 37 in high yields and excellent diastereo- and enantioselectivities by using quite unreactive $\pi$-electrophiles such as diethyl benzylidene malonate 36 [36]. Moreover, Jing, Qin, and co-workers reported an asymmetric synthesis of trans-configured trispirooxindoles by combining 35 and cyclic methyleneindolinones 38 in the presence of Takemoto's catalyst XIII (15 mol \%) [37]. Interestingly, less than $60 \mathrm{~min}$ is enough to the cascade reaction to reach completion and the expected spiro compounds that bears three quaternary stereocenters are isolated in good yield and selectivities.

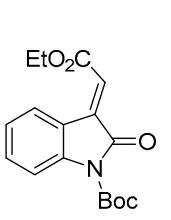

32<smiles>CC(C)(C)C1=CC(=Cc2ccccc2O)C=C(C(C)(C)C)C1=O</smiles>

33

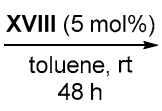

$48 \mathrm{~h}$

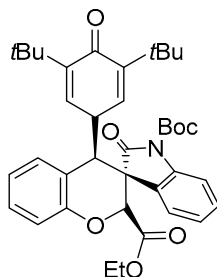

34

$89 \%$ Y

$(17: 1$ d.r., $99 \%$ ee $)$

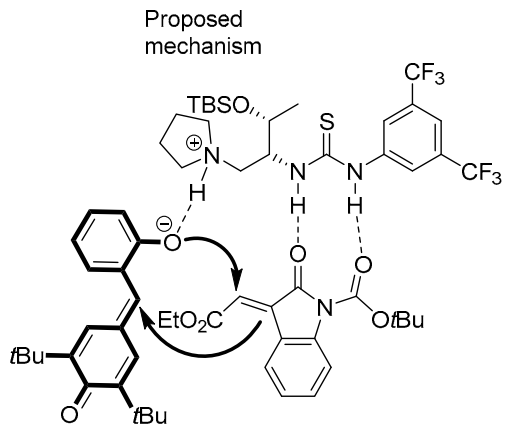

Scheme 8. Synthesis of chromans 34 with an oxindole moiety catalyzed by XVIII.

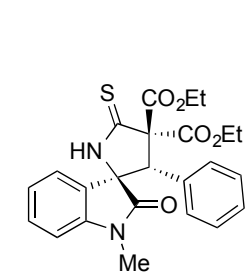

37

$78 \%$ Y $(97: 3$ d.r., $97 \%$ ee $)$<smiles>CCOC(=O)/C(=C\c1ccccc1)OCC</smiles>

XIX $(20 \mathrm{~mol} \%)$

toluene, $0{ }^{\circ} \mathrm{C}$

$10 \mathrm{~h}$

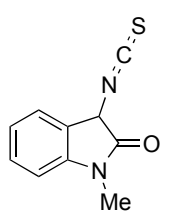

35

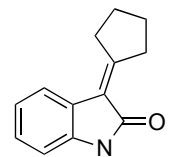

$38^{\text {Boc }}$

$\underset{\text { DCE, } 30{ }^{\circ} \mathrm{C}}{\stackrel{\text { XIII }(15 \mathrm{~mol} \%)}{\longrightarrow}}$ $40 \mathrm{~min}$

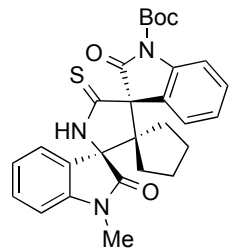

39

$84 \%$ Y (88:12 d.r., 93\% ee)

Scheme 9. 3-Isothiocyanate oxindoles as versatile substrates for the synthesis of densely functionalized spirooxindoles.

Remarkably, the use of chiral amino-thiocarbamate catalyst XXII (10 mol \%) gave rise to several polycyclic spirooxindoles 41 containing three contiguous chiral centers, with two of them having quaternary stereocenters, in the presence of 35 and 3-nitroindoles 40 (Scheme 10) [38]. Excellent yields and selectivitivites were obtained when the N1-position of 35 was blocked with a methyl group while slight erosion of the diastereo- and enantioselectivities were observed for more hindered N1-protecting groups.

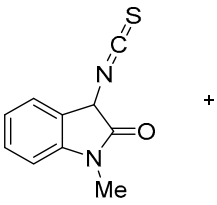

35<smiles></smiles>

40

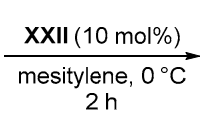

$2 \mathrm{~h}$

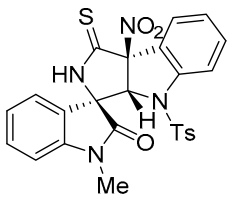

41

$99 \%$ Y $(98: 2$ d.r., $96 \%$ ee $)$

Scheme 10. Asymmetric synthesis of polycyclic spirooxindoles in the presence of amino-thiocarbamate catalyst XXII. 


\subsection{Michael Addition/Mannich/Cyclization Sequence}

Recyclable fluorous bifunctional Cinchona alkaloid catalyst XV [39] has proved its efficiency to catalyze cascade reaction in the presence of electron-deficient olefinic oxindole $\mathbf{4 2}$ and nucleophiles. The syntheses of spirooxindoles containing 2-piperidinone 44 and tetrahydropyridine 46 rings were successfully accomplished in 2015 by Zhang and co-workers through a four-component cascade transformation in the presence of diethyl malonate 43 or 1,3-diketone 45 respectively (Scheme 11) [40]. Under the optimal conditions [i.e., catalyst XV (10 mol \%) in toluene] high yields and levels of selectivity were reached affording polycyclic molecules densely functionalized that were prone to either further derivatization or scale-up. Although the authors have mainly proposed the use of oxindoles bearing a methyl group at the N1-position, one single example using free-N1 has been described affording slightly lower yields and comparable selectivities.
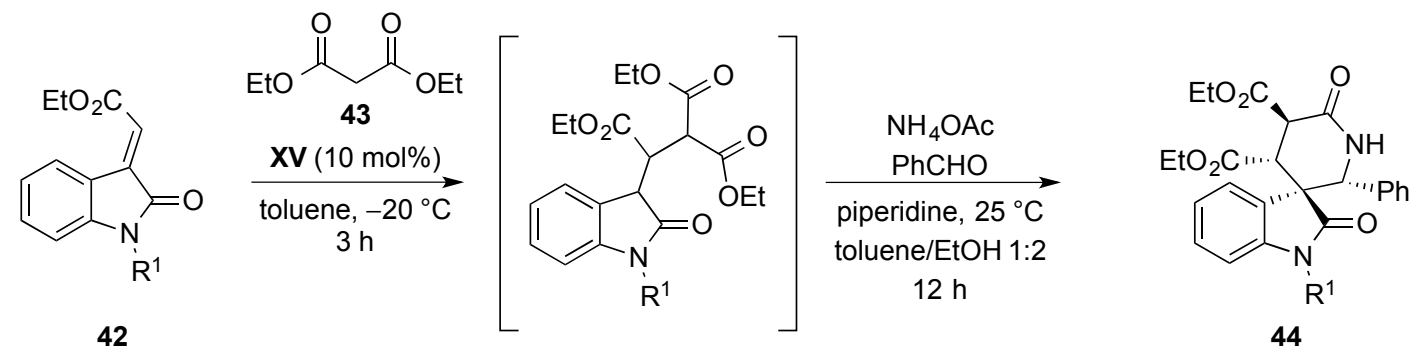

$\mathrm{R}^{1}=\mathrm{Me} ; 80 \% \mathrm{Y}(6: 1$ d.r., $98 \%$ ee $)$
$\mathrm{R}^{1}=\mathrm{H} ; \quad 60 \% \mathrm{Y}(6: 1$ d.r., $98 \%$ ee $)$<smiles>[R]N1C(=O)/C(=C/C(=O)OCC)c2ccccc21</smiles><smiles>CC(=O)C(C)=O</smiles>

42

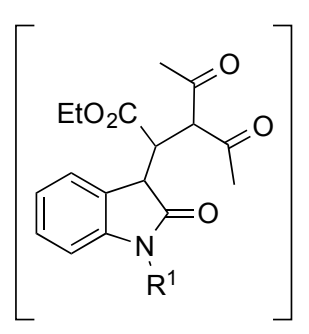

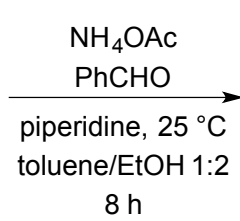

$8 \mathrm{~h}$<smiles>[R]N1C(=O)[C@@]2(c3ccccc31)[C@@H](c1ccccc1)NC(C)=C(C(C)=O)[C@H]2C(C)(C)C</smiles>

$\mathrm{R}^{1}=\mathrm{Me} ; 84 \% \mathrm{Y}(5: 1$ d.r., $96 \%$ ee $)$ $\mathrm{R}^{1}=\mathrm{H} ; 59 \% \mathrm{Y}(4: 1$ d.r., $94 \%$ ee $)$

Scheme 11. Synthesis of piperidone and tetrahydropyridine rings bearing spirooxindoles in the presence of recyclable catalyst XV.

Shortly after, the same group has used catalyst XV (10 mol \%) during a similar cascade reaction for the synthesis of spiro- $\gamma$-lactam oxindoles via a thiol-Michael/Mannich/lactamization cascade reaction in good yields and enantioselectivities and moderate diastereoselectivities [41]. The method goes through a four-component/one-pot synthesis and paves the way to novel compounds 48 containing three contiguous stereocenters including a quaternary one (Scheme 12). As for the previous example, $\mathrm{N}$-Me indoles were exclusively used as substrates and only one example with $\mathrm{N}-\mathrm{H}$ was reported. In both cases, catalyst recovery has been realized through first (i) loading onto a fluorous silica gel cartridge for solid-phase extraction (F-SPE) [42] followed by (ii) elution with 80:20 MeOH/ $\mathrm{H}_{2} \mathrm{O}$ for products and other non-fluorous components and $100 \% \mathrm{MeOH}$ for the catalyst $\mathrm{XV}$. Overall, catalyst is recovered in more than $91 \%$ yield and $>97 \%$ of optical purity. 

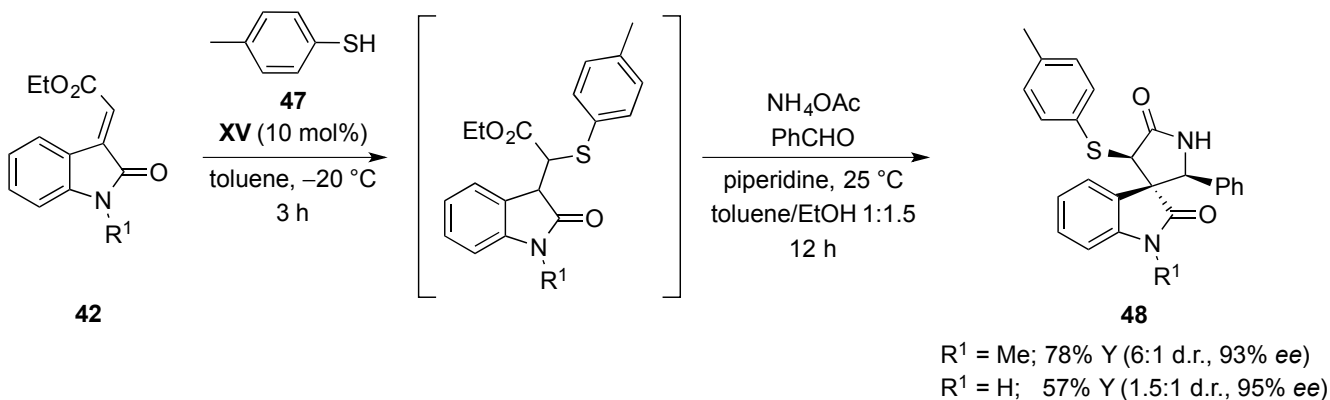

Scheme 12. Synthesis of spiro- $\gamma$-lactam oxindoles via cascade reaction catalyzed by catalyst XV.

Recently, Enders and co-workers have described the asymmetric preparation of trifluoromethylated 3,3'-pyrrolidinyl-dispirooxindole derivatives bearing four contiguous stereogenic centers among which two are vicinal [43]. The Michael-Mannich [3 + 2] cycloaddition takes place in the presence of oxindoles 42 and 49 through thiourea-based derivative XXI catalysis (Scheme 13). Concerning the scope, several olefinic oxindoles 42 (containing electron-neutral, electron-donating or electron-withdrawing groups in the benzene ring) as well as trifluoroethyl isatin ketimines 49 (bearing electron-donating or a 5-F groups in the benzene moiety) are well tolerated. To replace the Boc protecting group by a Me group on the nitrogen of substrate $\mathbf{4 2}$ did not hamper the enantioselectivities. Under the optimized conditions (i.e., XXI (10 mol \%) in $\mathrm{CCl}_{4}$ at $4{ }^{\circ} \mathrm{C}$ for $12 \mathrm{~h}$ ) the authors described 17 novel 3,3'-pyrrolidinlyl-dispirooxindoles 50 in good yields (60-92\%), moderate-to-good diastereoselectivities (4:1 to >20:1 d.r.) and enantioselectivities $(72-93 \% e e)$. In addition, scale up on a gram scale of the reaction was realized conserving high yields and keeping the same levels of selectivities.<smiles>O=C1/C(=N\CC(F)(F)F)c2ccccc2N1Cc1ccccc1</smiles>

49

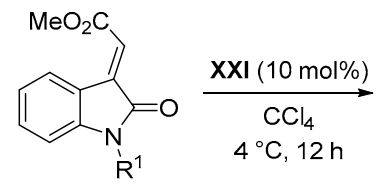

42
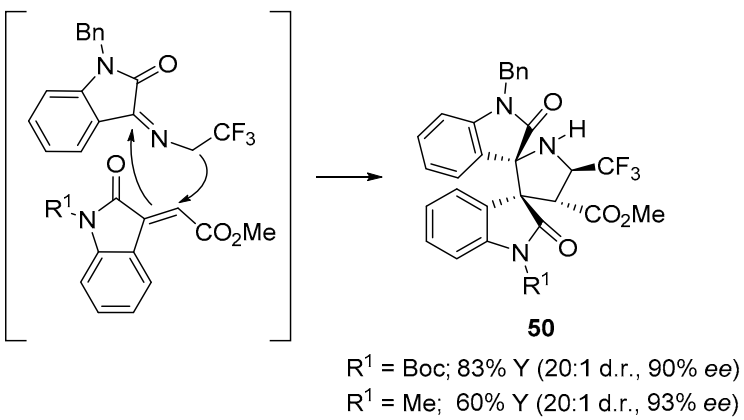

Scheme 13. Domino Michael-Mannich [3 + 2] cycloaddition for the asymmetric synthesis of 3,3'-pyrrolidinlyl-dispirooxindoles 50.

\subsection{Double Michael Addition Sequence}

A double Michael cascade reaction sequence, that allowed for a stereoselective [3+2] and [4+2] spiroannulation process, gave rise to both five- and six-membered $\beta$-nitro spirocarbocyclic oxindoles respectively [44]. After the screening of several thiourea-based organocatalysts, Quintavalla and co-workers have identified Takemoto's catalyst XIII (10 mol \%) as the most effective in terms of yields and selectivities. By combining 2-(2-oxoindolin-3-ylidene)-acetic esters $\mathbf{3 2}$ and nitroenoates 51 as donor/acceptor compounds, novel spirooxindoles densely functionalized were isolated via this Michael-Michael cascade process (Schemes 14 and 15). Noteworthy to mention, the authors foreground that upon varying the double bond geometry $(E$ or $Z)$ of nitroesters 51 , the configuration of the spiro quaternary stereocenter is inverted affording C3-epimers. This observation is valid for both five-(52) and six-membered (53) spirooxindoles. If the absolute configuration of the spiro center was determined accordingly to the $E / Z$ geometry of nitroesters double-bonds, the remaining stereocenters were forged under the catalyst XIII control. 


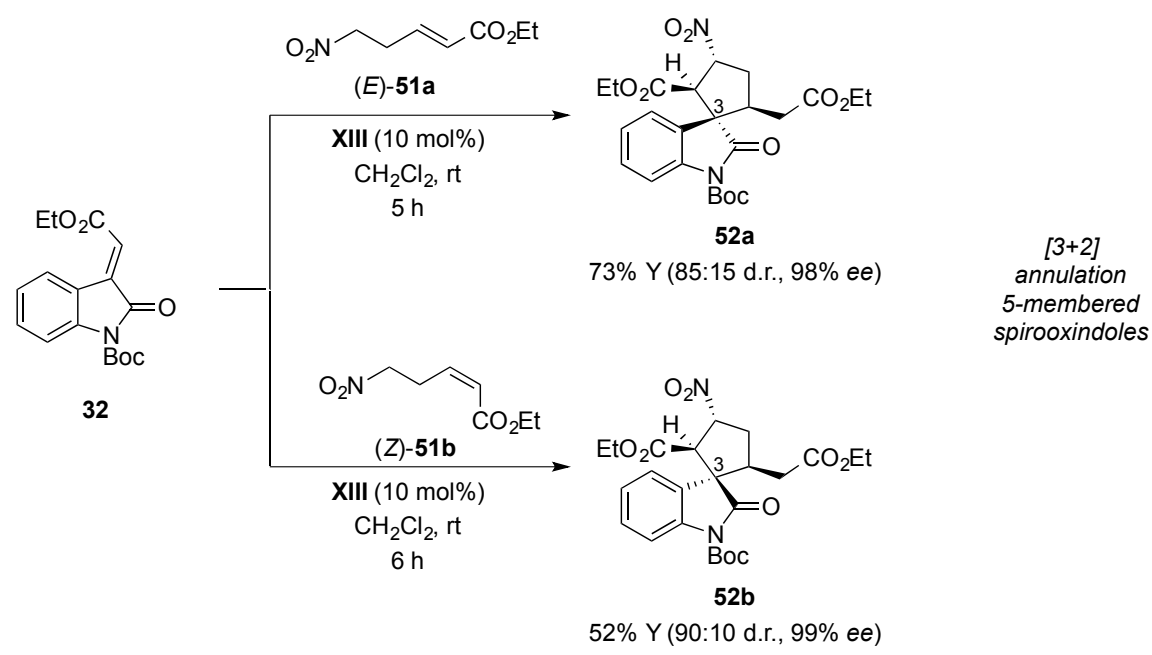

Scheme 14. Synthesis of $\beta$-nitro spirocyclopentane indolinones 52 via double Michael cascade in the presence of Takemoto's catalyst XIII.

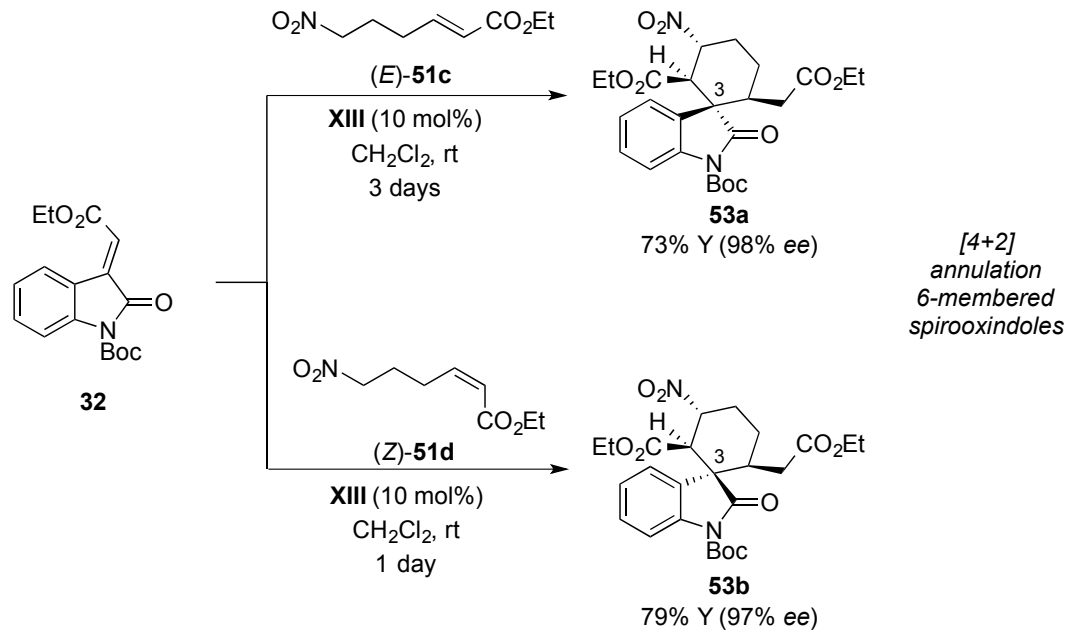

Scheme 15. Synthesis of $\beta$-nitro spirocyclohexane indolinones 53 via double Michael cascade in the presence of Takemoto's catalyst XIII.

\subsection{Aldol/Lactonization/Elimination Sequence}

An enantioselective domino reaction involving an unprecedented one-pot aldol/lactonization/elimination sequence has been developed, in 2016, yielding a broad range of 3 -spiro- $\alpha$-alkylidene- $\gamma$-butyrolactone oxindoles 58 (Scheme 16) [45]. The reaction is performed in the presence of $\beta$-nitro indolin-2-ones $\mathbf{5 4}$ and paraformaldehyde $\mathbf{5 5}$ as starting materials and it is catalysed by bifunctional Cinchona-derived thiourea XVI (10 mol \%) in dichloromethane at $0{ }^{\circ} \mathrm{C}$ or room temperature. While 54 was used as a 1:1 mixture of $C 3$ epimers, where both the $C \alpha$ and $C \beta$ absolute configuration were fixed and known [46], the expected products were isolated with well-established C3 quaternary spirocenter. Even though the two well defined $C \alpha$ and $C \beta$ stereocenters are destroyed during the domino process, it is interesting to mention that the only stereolabile C 3 center of 54 became the unique controlled and defined one present on the final products. It was postulated that the reaction might proceed through an aldol reaction between $\mathbf{5 4}$ and 55 to afford the acyclic intermediate 56 which bears three stereodefined centers including the fixed C3 quaternary one. Then, lactonization affords the cyclic lactone $\mathbf{5 7}$ which in turn loses its nitro group through $\mathrm{HNO}_{2}$ extrusion to afford the expected compounds 58 (Scheme 16). 


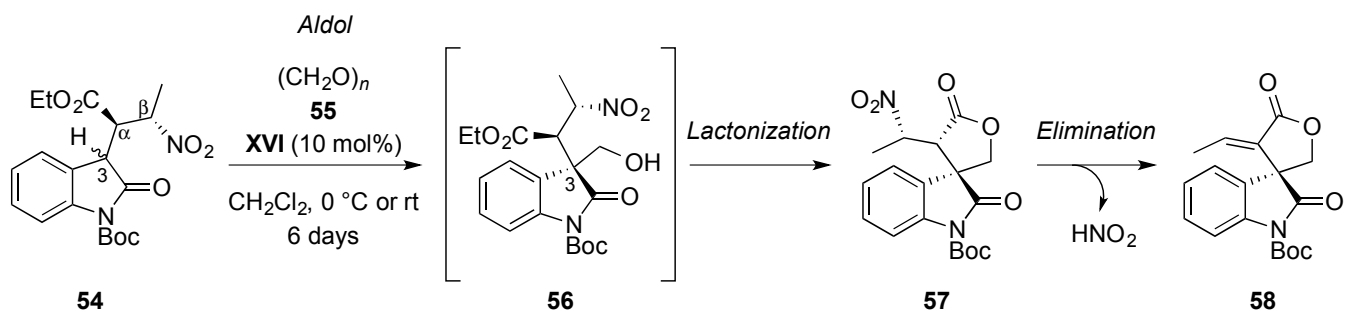

Scheme 16. Synthesis of 3-spiro- $\alpha$-alkylidene- $\gamma$-butyromactone oxindoles 58 in the presence of Cinchona-derived thiourea catalyst XVI.

\subsection{Friedel-Crafts/Hemiketalization Sequence}

The Kesavan group has published a straightforward synthesis of several oxindole-fused naphthopyran derivatives 60 by combining oxindole $\alpha$-ketoester 29 and 2-naphthol 59 and using a sequence of Friedel-Crafts-hemiketalization reactions [47]. Under the optimized conditions (i.e., XI (5 mol \%), in 1,1,1-trifluoromethyl benzene at room temperature) N1-protected oxindoles afforded the expected products in good yields and enantioselectivities. However, unprotected $\mathrm{N}-\mathrm{H}$ oxindoles conducted to lower yields and selectivities probably due to competitive binding of the $\mathrm{N}-\mathrm{H}$ site with the catalyst that might partially hamper the catalytic activity (Scheme 17).

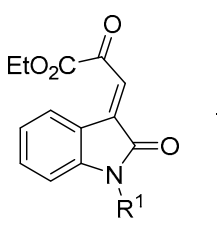

29

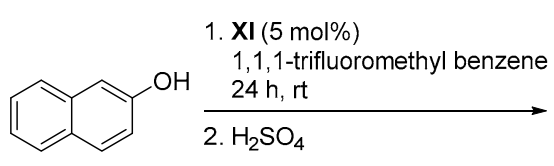

59

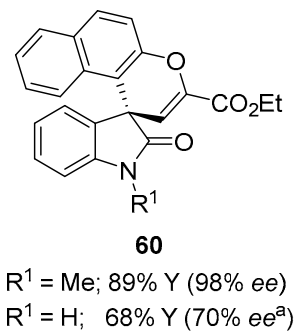

$\mathrm{R}^{1}=\mathrm{H} ; \quad 68 \% \mathrm{Y}\left(70 \% \mathrm{ee}^{\mathrm{a}}\right)$

Scheme 17. Synthesis of spirooxindole-naphthopyrans 60 in the presence of catalyst XI. a $86 \%$ ee after recrystallization.

\subsection{Miscellaneous}

The synthesis of spiro-3,4-dihydropyrans 62 bearing three stereocenters with vicinal quaternary ones has been reported by Kesavan and co-workers through the use of catalyst XI ( $5 \mathrm{~mol} \%)$ in toluene at room temperature (Scheme 18a) [48]. Supported by the obtained syn configuration of the secondary alcohol and the cyclopentanone moiety, the authors defend an inverse-electron-demand hetero-Diels-Alder reaction pathway of oxindole $\alpha$-ketoester 29 with cyclic $\beta$-oxoaldehyde 61 rather than a cascade transformation via Micheal addition/hemiketalization. An efficient enantioselective $[3+2]$ cyclization of 3-isothiocyanate oxindoles 63 and trifluoromethylated 2-butenedioic acid diester $\mathbf{6 4}$ or $\mathbf{6 5}$ paved the way to the synthesis of spirooxindoles with a $\mathrm{CF}_{3}$-containing all-carbon stereogenic center 66 (Scheme 18b) [49]. Interestingly, the authors highlighted the possibility to obtain either isomers (i.e., epimers at C4 position) in the presence of the same catalyst XIV ( $20 \mathrm{~mol} \%$ ) at different temperatures. This observation relies on the ability of isomerization of dimethyl maleate $\mathbf{6 4}$ into dimethyl fumarate 65 through azomethine ylide intermediate in the presence of the amine moiety of aminals [50]. 
(a)

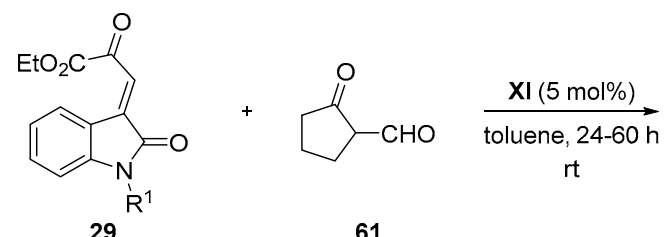

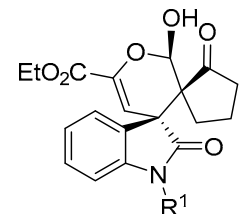

62

$\mathrm{R}^{1}=\mathrm{Me} ; 85 \% \mathrm{Y}(97 \%$ ee $)$

$\mathrm{R}^{1}=\mathrm{H} ; \quad 78 \% \mathrm{Y}(92 \%$ ee $)$

(b)<smiles>Cc1ccc2c(c1)C(N=C=S)C(=O)N2Cc1ccccc1</smiles>

63<smiles>CCOC(=O)C=C(C(=O)OCC)C(=CC(=O)OCc1ccccc1)C(F)(F)C(F)(F)F</smiles>

$\frac{\text { XIV }(20 \mathrm{~mol} \%)}{\text { toluene, } 12 \mathrm{~h}}$

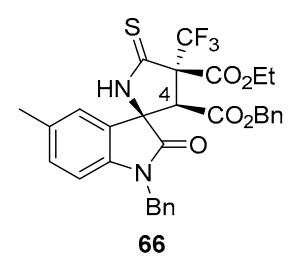

With 64: $94 \%$ Y $(20: 1$ d.r., $94 \%$ ee $)$ With 65 : $90 \%$ Y $(15: 1$ d.r., $92 \%$ ee $)$

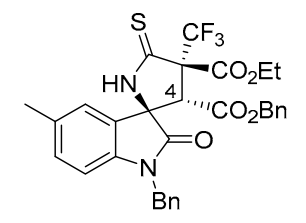

66

$94 \%$ Y $(20: 1$ d.r., $93 \%$ ee $)$

Scheme 18. (a) Synthesis of spiro-3,4-dihydropyrans 62 and (b) synthesis of trifluoromethylated spirooxindoles derivatives 66 .

\section{Squaramide Catalysts}

Inspired by the ability of urea/thiourea derivatives to promote high stereoselective organocatalytic reactions, several research groups have been engaged in the construction of alternative and complementary catalysts that involve in their architecture less explored H-bond donor motifs. Among others, in 2008 Rawal and co-workers postulated the potential of squaramide catalophores [51]. Specifically, over the years, several thorough studies have recognized that secondary squaramides featured by two $\mathrm{H}$-bond donors $(\mathrm{NH})$ and two $\mathrm{H}$-bond carbonyl acceptors $(\mathrm{C}=\mathrm{O})$ should easily establish a strong hydrogen bond network with acceptors and donors as well as with mixed acceptor-donor systems (Figure 5a).

Additionally: (i) the conformational restriction due to the aromaticity enhancement of the cyclobutendione core upon the delocalization of nitrogen lone pairs, which make the two N-H bonds coplanar with the rigid "squara structure" and (ii) the distance between the two N-H that is of $0.6 \AA$ broader than in thioureas, make squaramide derivatives not only a valuable alternative to the urea/thiourea counterpart but also a class of more wide-range applicable organocatalysts (Figure $5 b$ ).

Herein we are going to depict selected recent examples that demonstrate their remarkable ability in various cascade reactions involving an oxindole derivative as substrate and the catalophores depicted below (Figure 6). 
(a) H-Bond Interactions
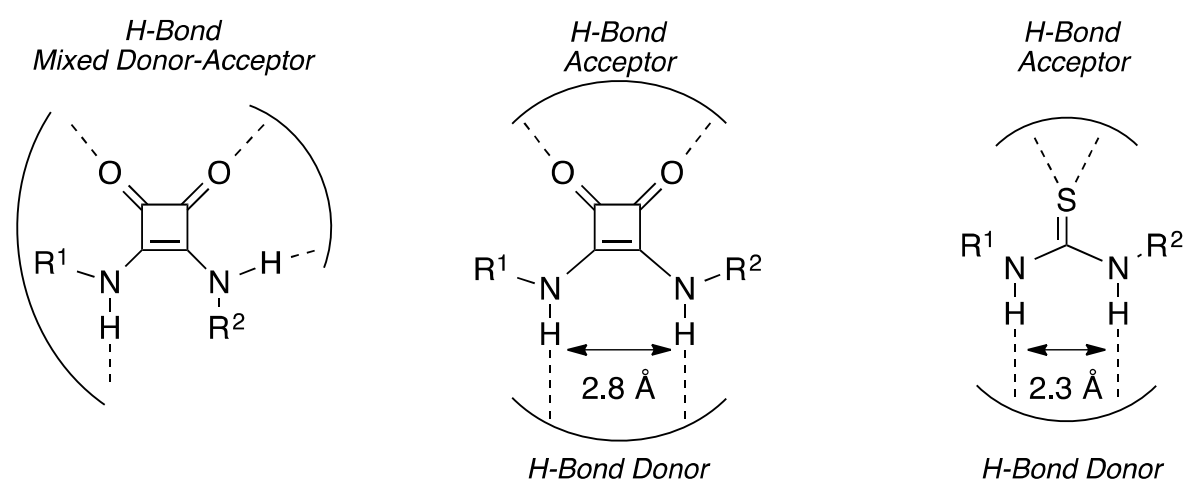

(b) Resonance forms of the aromatic system

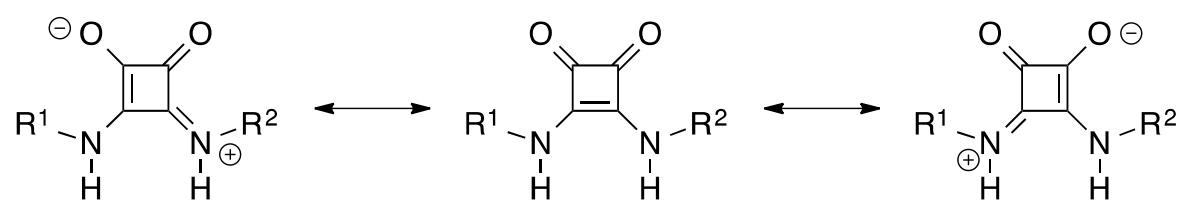

Figure 5. (a) H-Bond donor ability of squaramide and thiourea; (b) Lone pairs conjugation and resonance forms of the aromatic system.

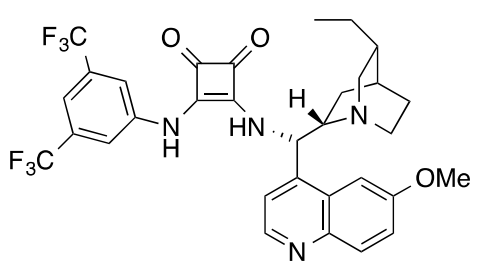

XXIII<smiles>C=CC1CC2CCN1CC2c1ccnc2ccccc12</smiles>

XXVI

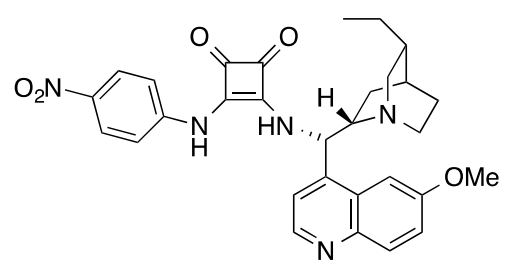

XXIV

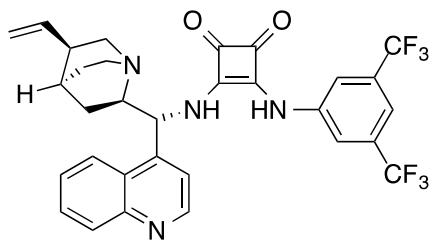

XXV<smiles></smiles>

XXVIII<smiles></smiles>

XXIX

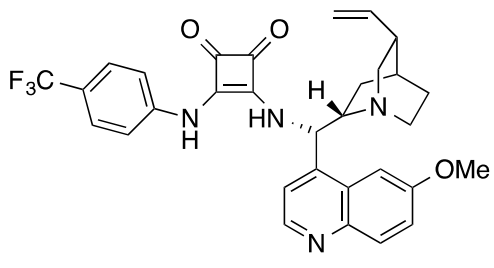

XXVII

Figure 6. Squaramide based organocatalysts reported in the present section. 


\subsection{Double Michael Addition Sequence}

As stated before, oxindole derivatives have been a privileged substrate in organocatalysis. Specifically, as consequence of its unique electron-demand, $\alpha$-alkylidene oxindoles have been often involved in cascade reactions mainly relying on Michael addition promoted by both C-nucleophiles and hetero atoms. In such a scenario, squaramide derivatives clearly have ever played a predominant role since their undiscussed ability as bifunctional catalysts [52]. Notably in this field Zhao and Du devoted their efforts not only to the design and synthesis of novel more efficient squaramide organocatalysts but also to the preparation of uncommon cascade reagents that should be suitable to build quite complex and densely functionalized carbocycles. Thus, in 2015, their group reported the first squaramide asymmetric protocol for the synthesis of chiral spiro[pyrrolidine-3,3'-oxindole]s 67 (Scheme 19). The optimized cascade aza-Michael/Michael addition sequence, distinguished for its mild conditions (i.e., $-10^{\circ} \mathrm{C}$ in $\left.\mathrm{CH}_{2} \mathrm{Cl}_{2}\right)$ and quite low catalyst loading $(5 \mathrm{~mol} \%$ ), was easily applied to a broad substrate scope achieving the desired products 67 in high yields with excellent diastereo(up to $>99: 1$ d.r.) and enantioselectivities (from $93 \%$ ee to $>99 \%$ ee in almost all cases).<smiles>[R]OC1CN([18O])C(=O)C12C(=O)N([R6])c1ccc([R])cc12</smiles>

67

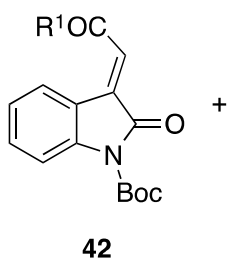

42

$\mathrm{R}^{1}=\mathrm{OEt} ; \mathrm{R}^{2}=\mathrm{Ph}$

$\mathrm{R}^{1}=\mathrm{Ph} ; \mathrm{R}^{2}=\mathrm{Me}$

$\mathrm{R}^{1}=\mathrm{OBn} ; \mathrm{R}^{2}=\mathrm{Ph}$

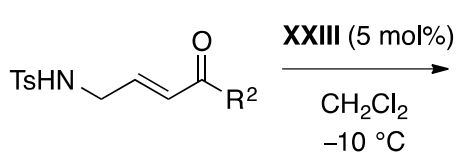

68

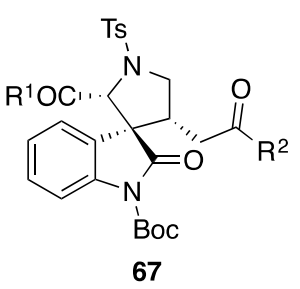

$99 \%$ Y (95:5 d.r.; $95 \%$ ee $)$

$72 \%$ Y $(96: 4$ d.r.; $98 \%$ ee $)$

$96 \%$ Y (96:4 d.r.; $96 \%$ ee $\left.{ }^{a}\right)$

Scheme 19. Asymmetric cascade aza-Michael/Michael addition for spiro[pyrrolidine-3,3'-oxindoles]s 67.

a The reaction was carried out on gram scale.

Remarkably, the reaction could be repeated even on gram scale without any loss in both yield or stereoselectivity. The authors reasoned that such an outstanding stereocontrol should be ascribed to the squaramide moiety that contemporarily activates and orients the (E)-tert-butyl 3-(2-ethoxy-2-oxoethylidene)-2-oxoindoline-1-carboxylate 32 as well as the hydroquinine framework and enhances the nucleophilicity of the tosylaminomethyl enone 68 (Scheme 20). In the aza-Michael addition, the settled H-bond network (A) drives the nitrogen attack only from the Si face of the substrate to furnish the intermediate $\mathbf{B}$ where the enone group undergoes an intramolecular Michael addition on the $S i$ face via the transition state $\mathbf{C}$, which rapidly provides the product 67 and restores the catalyst XXIII.

The just disclosed reaction mechanism and the consequent stereochemical outcomes prompted the authors to replace the $\alpha, \beta$-unsaturated esters with $\alpha$-alkylidene succinimides 69 structurally similar to the oxindole counterpart [53]. The previously developed squaramide-catalysed approach in even milder conditions (i.e., room temperature and THF as solvent) guaranteed the formation of highly functionalized spirooxindoles $\mathbf{7 0}$ in elevated yield and stereocontrol (up to $97: 3$ d.r., up to $98 \% e e$ ). In Scheme 21 it is reported the most representative examples of the produced library, which was also synthesized in gram scale without any loss in term of stereoinduction. 


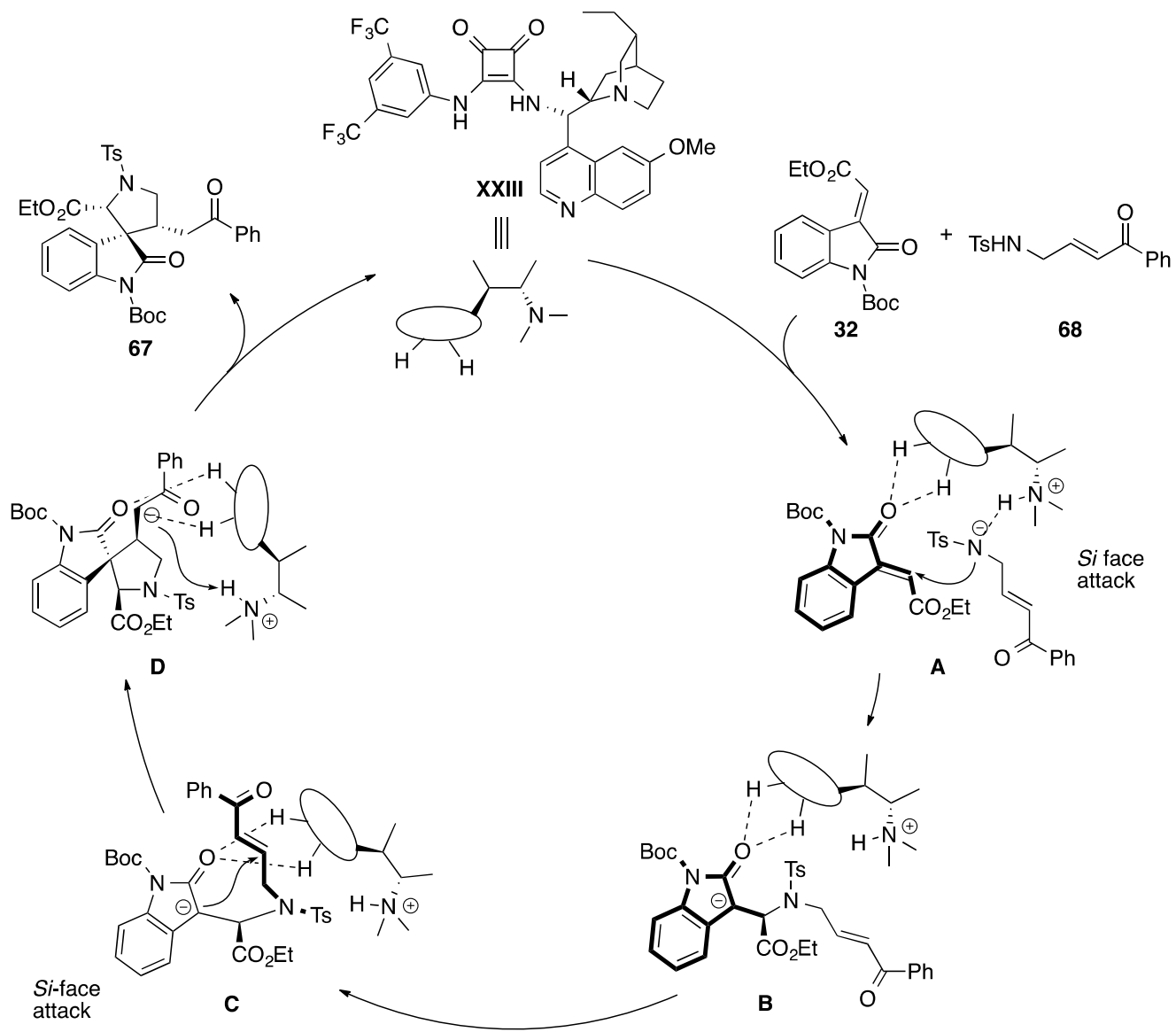

Scheme 20. Proposed reaction mechanism for the aza-Michael/Michael cascade reaction developed by Zhao and Du. A-D are hypothesized intermediates on the catalytic proposed cycle<smiles>[R20]C/C=c1\c2cc[R1]([H])ccc=2n(C(C)=O)c1=O</smiles>

42<smiles>[R9]C=C1CC(=O)N(C(C)(C)C)C1=O</smiles>

69

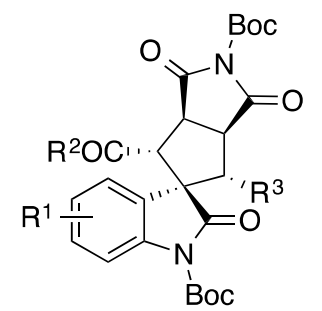

70

$$
\begin{aligned}
& \mathrm{R}^{1}=\mathrm{H} ; \mathrm{R}^{2}=\mathrm{Et} ; \quad \mathrm{R}^{3}=\mathrm{Ph} \\
& \mathrm{R}^{1}=\mathrm{H} ; \mathrm{R}^{2}=t \mathrm{Bu} ; \mathrm{R}^{3}=3-\mathrm{MeOC}_{6} \mathrm{H}_{4} \\
& \mathrm{R}^{1}=\mathrm{H} ; \mathrm{R}^{2}=t \mathrm{Bu} ; \mathrm{R}^{3}=\mathrm{Ph}
\end{aligned}
$$

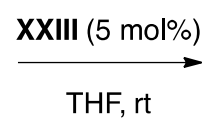

$83 \%$ Y (86:14 d.r.; $98 \%$ ee $)$ $86 \%$ Y $(95: 5$ d.r.; $94 \%$ ee $)$ $85 \%$ Y $\left(92: 8\right.$ d.r.; $\left.91 \% e e^{a}\right)$

Scheme 21. Organocatalytic cascade Michael/Michael addition for spirooxindoles containing five contiguous stereocenter. ${ }^{\text {a }}$ The reaction was carried out on gram scale.

Once validated the efficiency of their squaramide XXIV as catalyst, in 2016 Zhao and Du designed the new cascade reagent $\mathbf{7 2}$ featured by both an active nucleophile center and an electrophile site (Scheme 22) [54]. The authors demonstrated their hypothesis fruitfully employing the developed donor Michael acceptor reagent 72 in a tandem Michael/Michael reaction which smoothly furnished highly functionalized bispirooxindole-tetrahydrofurane scaffolds 73 bearing four contiguous stereocenters. Despite the number of different substituents introduced on each oxindole framework, the overall 
process never failed to give the expected products 73 in very high levels of optical purity ( $>20: 1$ d.r., up to $>99 \%$ ee) and moderate-to-high yields ( $58-96 \%$ yield).
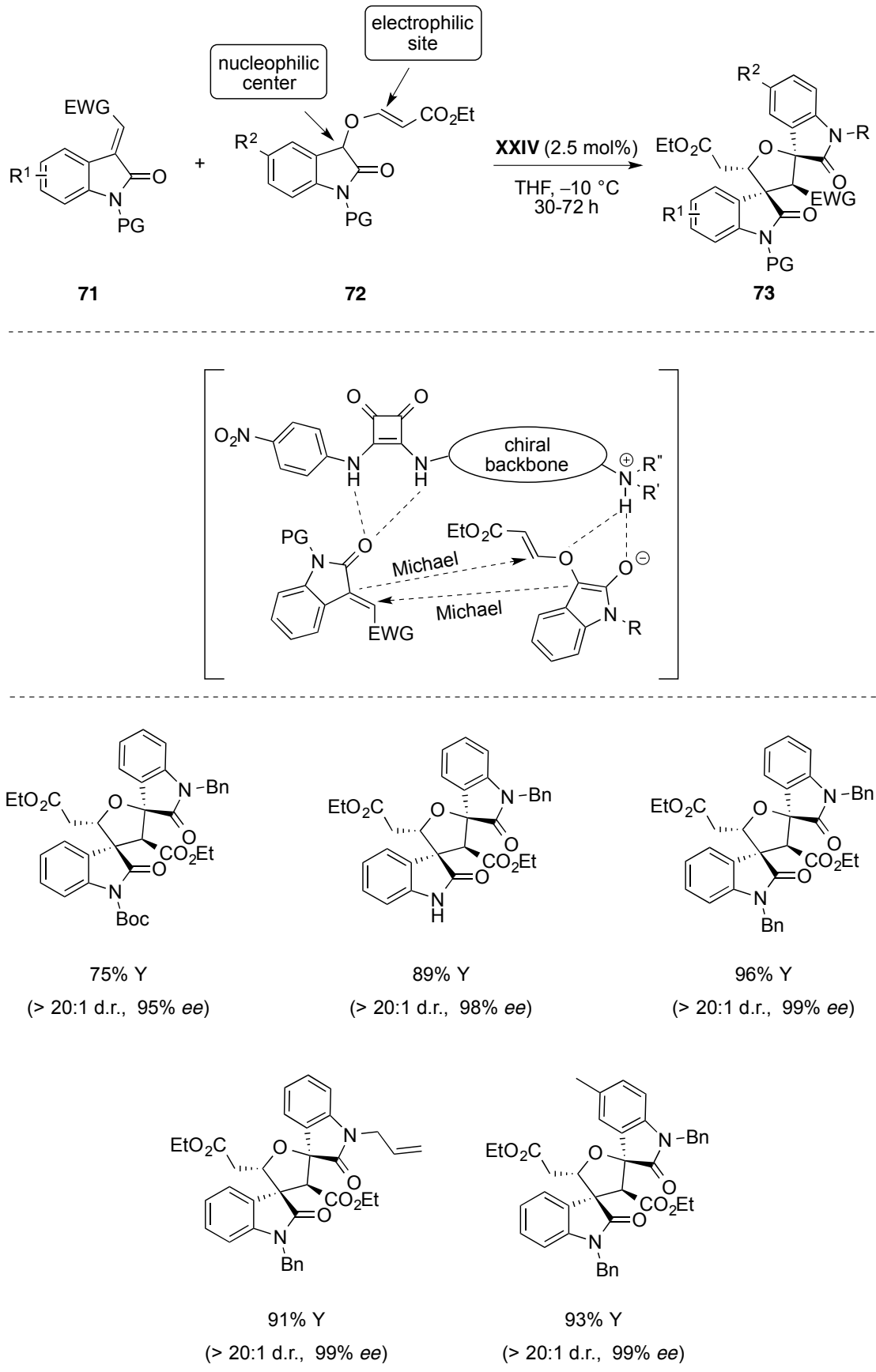

Scheme 22. Asymmetric enantioselective tandem Michael/Michael reaction to bispirooxindoles 73.

\subsection{Michael Addition/Cyclization Reaction Sequence}

A second highly exploited reaction sequence that straightforwardly furnishes complex spiro compounds involves a Michael addition followed by a fast cyclization reaction (see also Section 4.1). Among all the possible substrate/reagent systems largely reported in literature, Yuan and co-workers chose the previously investigated 3-hydroxyoxindoles 75 and 3-aminooxindoles $\mathbf{7 6}$ as initial Michael donor and the $\alpha, \beta$-unsaturated acylphosphonates as Michael acceptor [55]. Such an uncommon electron poor counterpart was mainly selected due to the lability of the C-P bond that simplifies the phosphonate group removal. Relying on the efficiency of squaramide organocatalyst XXV 
together with the designed starting molecules, the authors developed a highly stereoselective Michael/cyclization cascade sequence able to afford a broad spectrum of spirocyclic oxindoles 78 and 79. Indeed, applying the initial optimized conditions (i.e., $\mathrm{CH}_{3} \mathrm{CN}$ or $\mathrm{CH}_{2} \mathrm{Cl}_{2}$ as solvent, room temperature), the overall reaction proceeded without any loss in stereoslectivity (up to $>99: 1$ d.r.; $71-97 \% e e$ ) and yield (up to $98 \%$ ) when (i) the Michael donor was either a 3-hydroxy or a 3-aminooxindoles ( 75 and 76 respectively), which afford to the spiro $\gamma$-lactones ( 78 for $X=O$ ) and $\gamma$-lactames ( 79 for $X=N$ ), as well as (ii) substituents quite different in terms of electron demand and steric hindrance were introduced on both nitrogen and aromatic ring.

A slight drop in the stereoinduction was observed when the $\beta$ position of the $\alpha, \beta$-unsaturated acylphosphonate 74 was decorated with excessively bulky groups (Scheme 23). The observed efficiency should be due to the dual activation of the catalyst that (i) promotes the enolyzation of the 3-hydroxyoxindoles and (ii) exposes the Re face of the $\alpha, \beta$-unsaturated acyl phosphonate to the attack from the $\mathrm{Si}$-face of the just generated nucleophile.

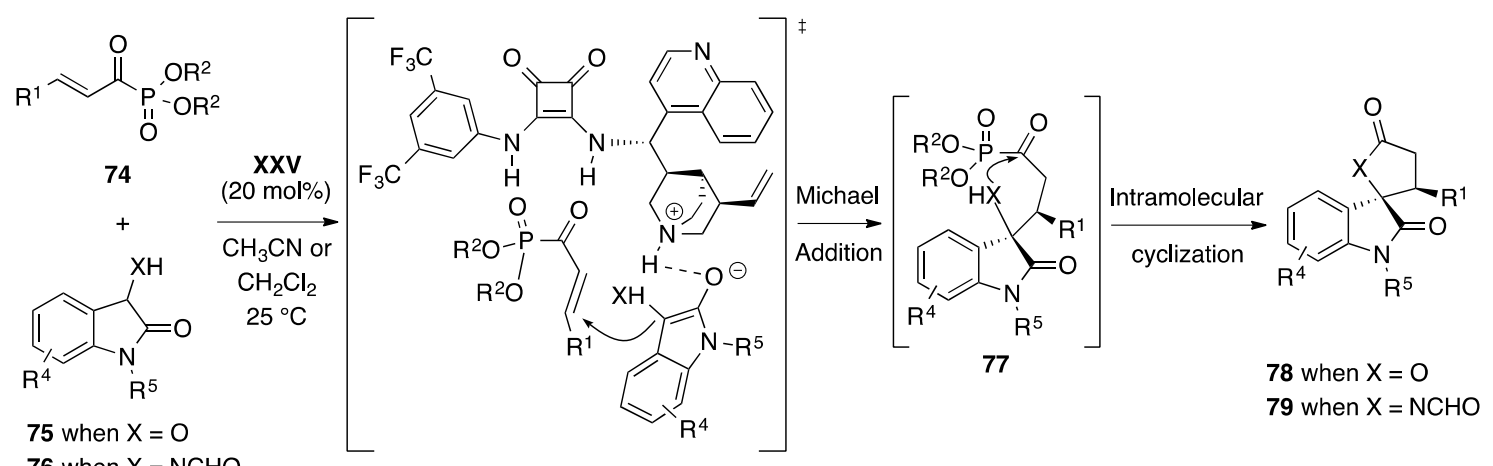

76 when $\mathrm{X}=\mathrm{NCHO}$

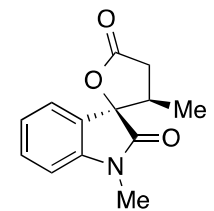

$70 \% Y$

(> 99:1 d.r., $94 \%$ ee)

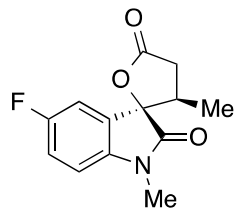

$89 \% Y$

(> 99:1 d.r., 95\% ee)

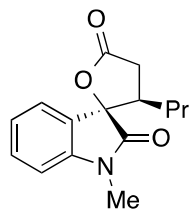

$94 \% Y$

(98:2 d.r. $95 \%$ ee)

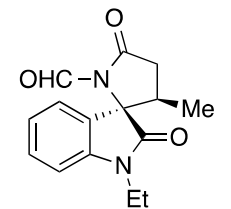

$49 \%$ Y

(> 99:1 d.r., $97 \%$ ee)

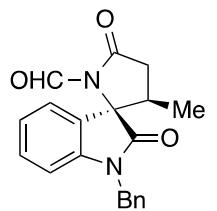

$65 \% Y$

(98:2 d.r., $94 \%$ ee)

Scheme 23. Organocatalysed Michael/cyclization cascade reaction for the construction of spirocyclic oxindole- $\gamma$-lactones /lactams.

More recently, the similar squaramide-quinine based catalyst XXVI (10 mol \%) was effective when $\alpha, \beta$-unsaturated $N$-acylated succinimides $\mathbf{8 0}$ were chosen as the initial Michael acceptor and 3-hydroxyoxindoles 75 as the nucleophilic counterpart (Scheme 24). [56] Specifically, after having carefully optimized the reaction conditions (i.e., $\mathrm{CH}_{2} \mathrm{Cl}_{2}$ as solvent, $-10{ }^{\circ} \mathrm{C}$ as best temperature), $\mathrm{Du}$ and co-workers validated their protocol by performing the proposed asymmetric cascade Michael/cyclization reaction on various Michael donor/acceptor systems obtaining the corresponding spirooxindoles lactones $\mathbf{7 8}$ in good yields (75-89\%). Although the stereochemical outcomes were always excellent in term of enantioselectivity $(96-99 \% e e)$, the diastereomeric ratios seemed to be more easily affected by various substituents introduced both on the enone system and on the oxindole moiety (from 75:25 to $>95: 25$ d.r.). Afterwards, once assigned the correct configuration to the generated stereocenter, the authors provided a mechanistic study that pointed out how the quinine-derived squaramide XXVI should act as bifunctional catalyst. As depicted in Scheme 24, the tertiary amine unit deprotonates the 3-hydroxyoxindole while the squaramide moiety binds the resulting nucleophile (A) which in turn, attacks the electrophile already activated by the protonated amine (B). The Michael 
adduct $\mathbf{C}$ undergoes an intramolecular cyclization which removes the succinimide auxiliary and generates the expected spirooxindole 78 .

Exploiting the well-known reactivity of 3-isothiocyano oxindoles conferred by the strongly electron withdrawing NCS group, $\mathrm{Du}$ and co-workers reported also the preparation of more complex pyrrolidinyl spirooxindoles employing either chalcones 82 [57] or maleimides 84 [58] as acceptor counterpart (Scheme 25). In both cases the extremely similar quinine-derived squaramide XXVII (10 mol \%) and XXVIII ( $5 \mathrm{~mol} \%$ ) resulted to be the best catalysts in the same reaction condition (i.e., $\mathrm{CH}_{2} \mathrm{Cl}_{2}, 0{ }^{\circ} \mathrm{C}$ ). The desired spirocyclic products 83 and 85 were achieved in high yields (87-99\%) and excellent diastereo(up to $>99: 1$ d.r.) and enantioselectivities (up to $99 \%$ ee) triggered by a complex H-bond network where the organocatalyst enable the stereocontrolled attack of the oxindole donors toward either the electron-poor chalcones $\mathbf{8 2}$ or maleimides $\mathbf{8 4}$.<smiles>O=C1C(O)C2=C(C=C[Se]C=C2)N1P(=O)([O-])O</smiles>
75<smiles>[R]C=CC(=O)N1C(=O)CCC1=O</smiles>
80

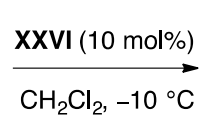

$\mathrm{CH}_{2} \mathrm{Cl}_{2},-10{ }^{\circ} \mathrm{C}$

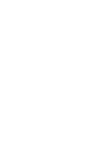

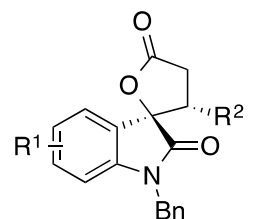

78

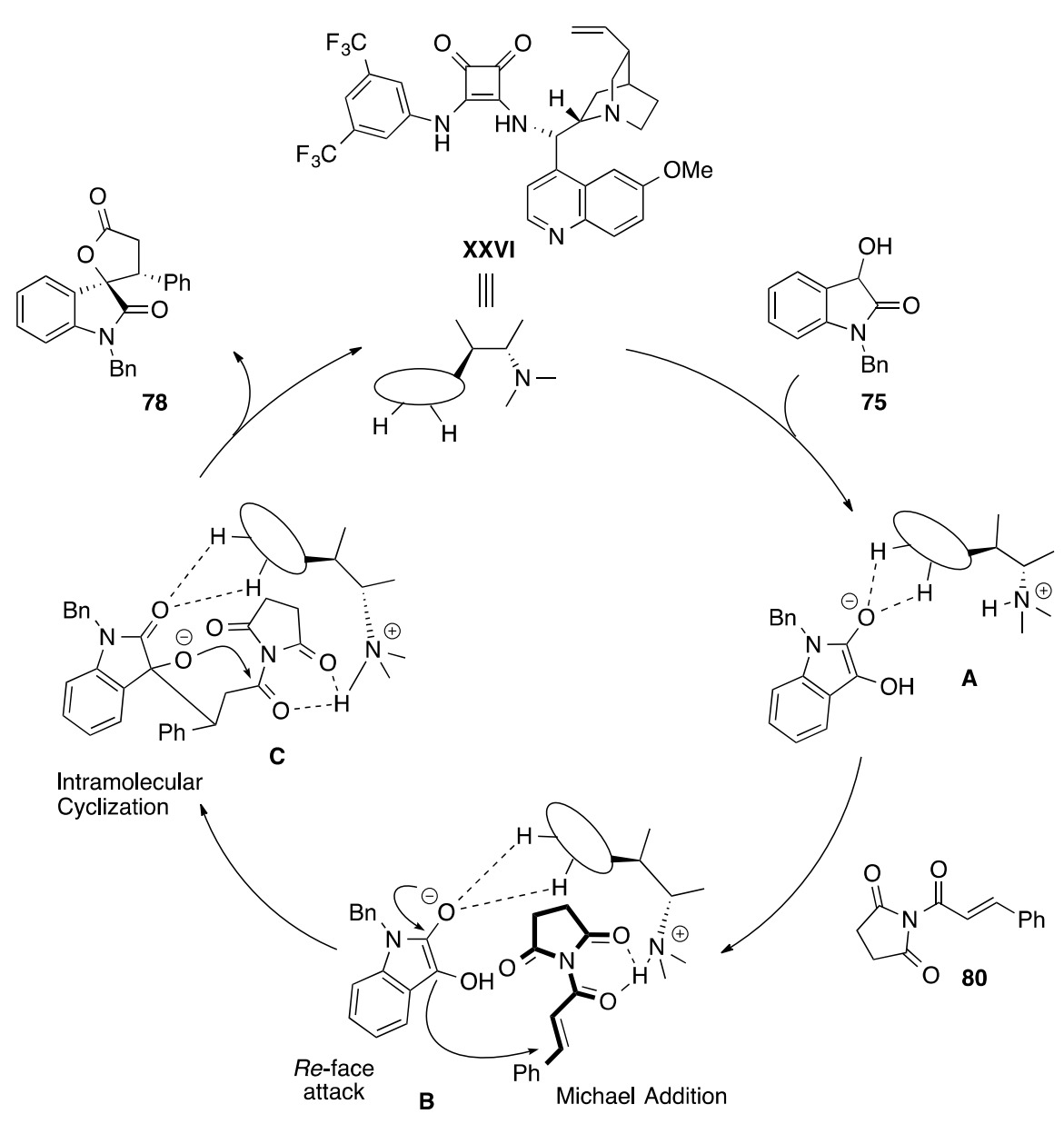

Scheme 24. Asymmetric cascade Michael/cyclization reaction performed on 3-hydroxyoxindoles/ $\mathrm{N}$-acylated succinimides as donor/acceptor system. A-C are hypothesized intermediates on the catalytic cycle proposed. 


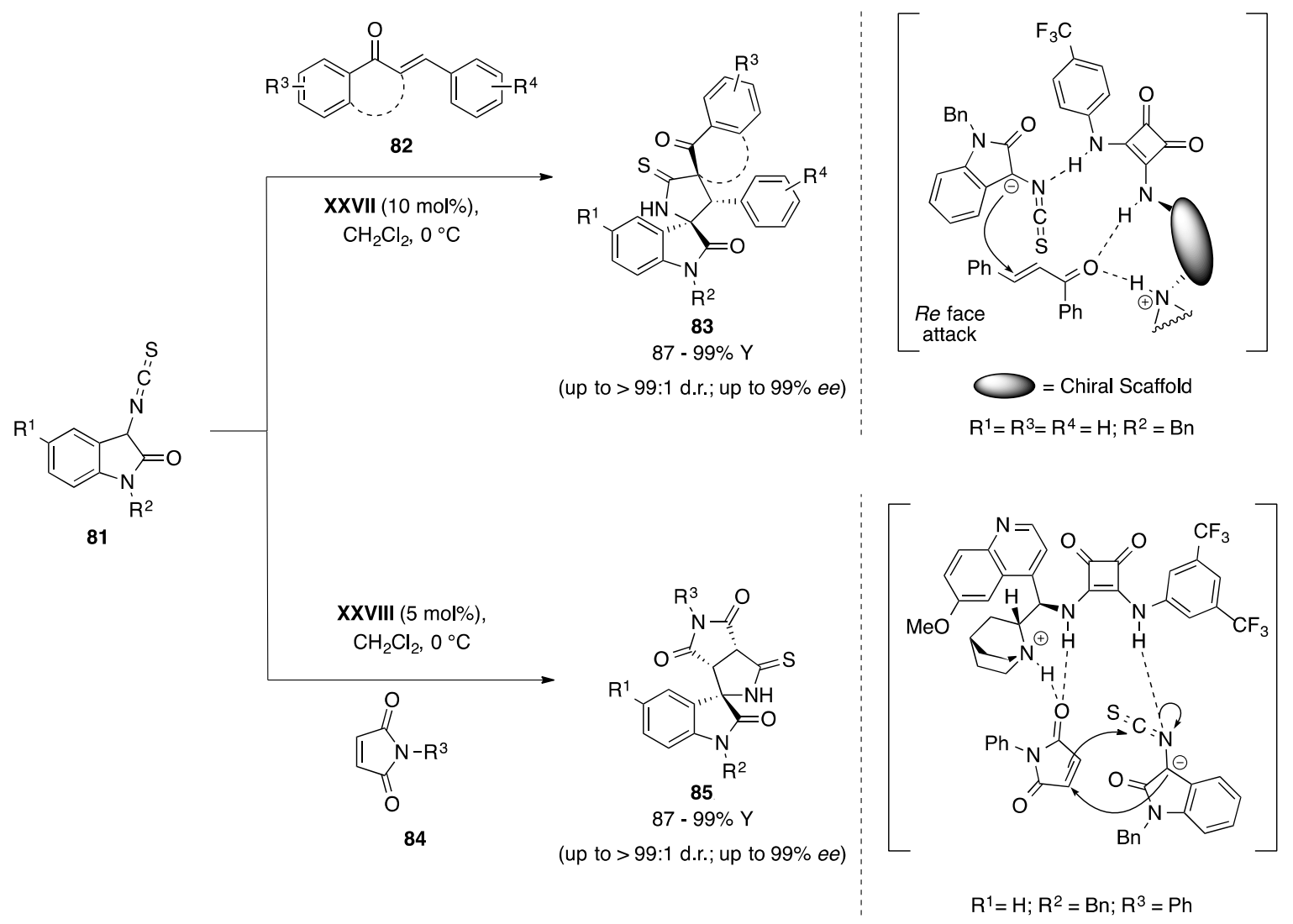

Scheme 25. Du's research toward the preparation of pyrrolidinyl spirooxindoles 83 and 85 employing respectively chalcones 82 and maleimides 84 .

The substitution of quinine moiety with a tertiary ethylenediamine framework decorated with naphthyl groups opened the door to the synthesis of pharmacologically interesting dihydropyranoindole derivatives. Indeed, Zhao and co-workers performed a Michael addition/cyclization sequence at relatively low temperature $\left(-20^{\circ} \mathrm{C}\right)$ in $\mathrm{CH}_{2} \mathrm{Cl}_{2}$ employing the squaramide derivative XXIX as organocatalyst able to join together the isatine-based trifluoroacrylates 86 and the malonitrile 15 (Scheme 26) [59]. The hypothesized products 87, bearing a novel trifluoromethylated all-carbon-substituted stereocenter, were almost always isolated with excellent yields (91-99\%) and high level of optical purity (86-99\% ee). Unfortunately, the optimized protocol failed not only when less hindered substituents $\left(R^{2}=\mathrm{Me}, \mathrm{Ac}\right)$ were introduced as protecting groups in the oxindole unit, but also when the malonitrile 15 was replaced with the less reactive ethyl cyanoacetate 88. Noteworthy, in the latter case, nearly analogous outcomes in term of yields (38-79\%) and stereocontrol (85-98\% ee) were accomplished only when the squaramide catalyst XXIX was substituted with the $(1 R, 2 R)$-diphenyl-1,2-diamine derived bifunctional thiourea XII.

The just depicted results, one more time, confirm the complementary use of thiourea-based and squarate derived organocatalyst for the stereoselective preparation of complex bioactive products. 


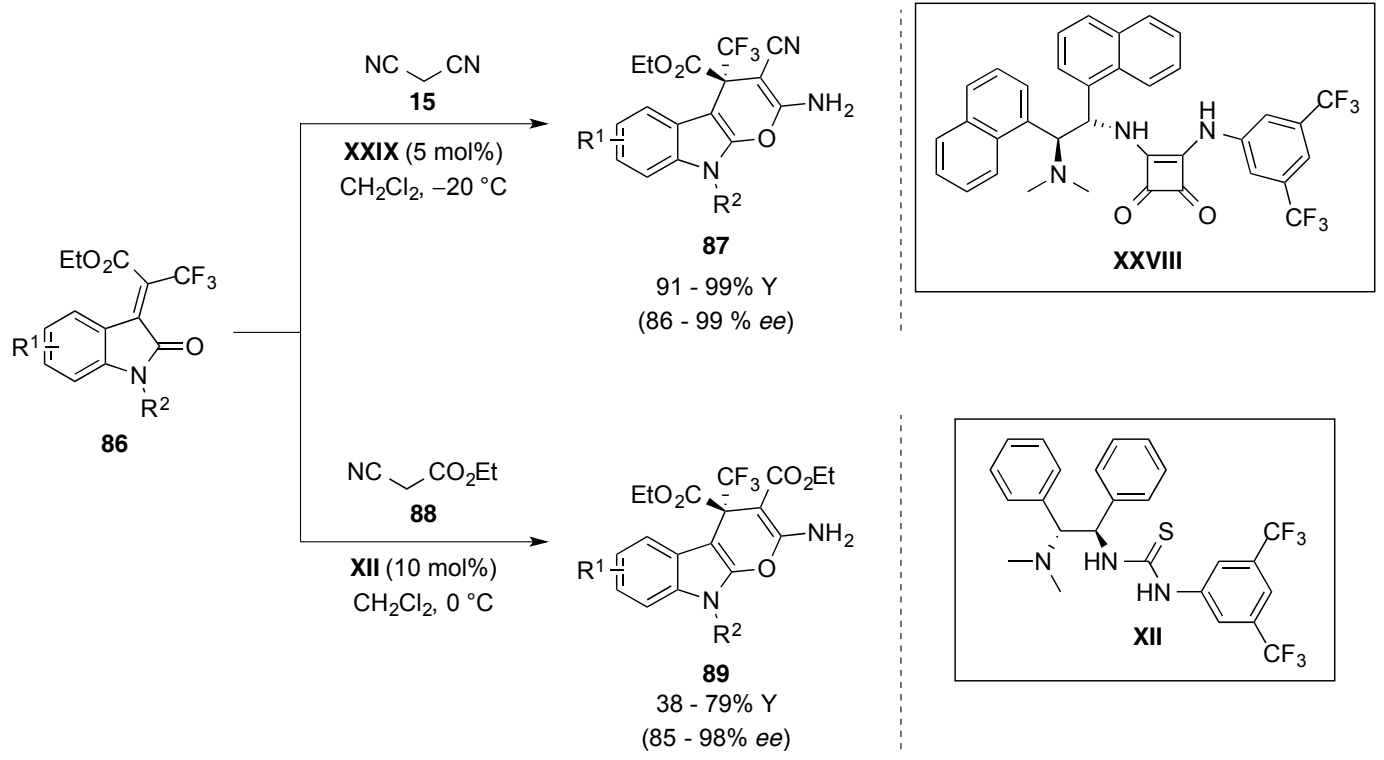

Scheme 26. Synthesis of dihydropyranoindole derivatives, a comparison between squaramide-based and thiourea catalyst.

\subsection{Miscellaneous}

In 2012 Casiraghi and co-workers illustrated how 3-alkylidene oxindoles could also react as vinylogous nucleophile in order to functionalize the $\gamma$-position in the presence of a suitable electrophile [60,61]. Inspired by this evidence as well as by subsequent Casiraghi's researches concerning the Mukaiyama type aldol reactions [62], Han and Chang postulated the creation of biologically relevant 3-hydroxyoxindole framework relying on the nucleophilicity of 3-alkylidenes 90 and the undeniable electrophilicity of isatins 91 [63]. Surprisingly, instead of obtaining the expected aldol adduct, the spirooxindole dihydropyranones 93 were isolated by using the chiral Cinchona alkaloid-squaramide bifunctional organocatalyst system XXVIII (Scheme 27). The stereochemical outcome of the overall reaction was not influenced by the introduction of different substituents (i.e., in terms of either electron demand or steric hindrance) on both substrates (87-99\% ee). A slight drop down was observed in the yield only when a $\mathrm{NO}_{2}$ group decorated the electrophilic aromatic ring $\left(\mathrm{R}^{3}=\mathrm{NO}_{2}\right)$. Without going in fine details, the authors suggested two models for explaining the dual activation of both nucleophile and electrophile (Scheme 27, B and C) which could account for the Si face addition of the s cis-enolate in the initial aldol reaction. Once obtained the intermediate $\mathbf{A}$, the surprising replacement of the lactam $\mathrm{C}-\mathrm{N}$ bond with a lactone $\mathrm{C}-\mathrm{O}$ bond occurs delivering the product 93 after protonation and catalyst regeneration.

Such an uncommon behaviour of amide C-N bond was also recently detected by Zhao and co-workers during their studies concerning the organocatalytic Friedel-Crafts/lactonization domino reaction (Scheme 28) [64]. Actually, the authors reported a remarkable example of the employment of squaramide catalophore $\mathbf{X X X}$ in the asymmetric synthesis of dihydrocoumarins, a ubiquitous scaffold in bioactive natural products. Specifically, the formal [3+3] protocol involved an initial Friedel-Crafts alkylation of naphthols at the $C_{\beta}$ position of the 3-ylidene oxindoles 94 (A, Scheme 28) followed by an unexpected intramolecular cyclization with a $\mathrm{C}-\mathrm{N}$ bond cleavage of the lactam moiety by the phenolic hydroxyl group (B, Scheme 28). 


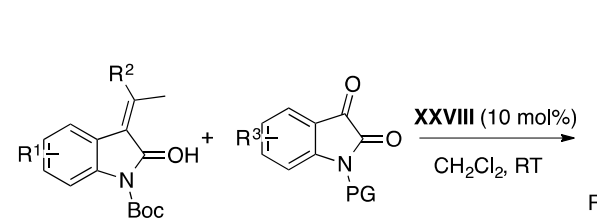

90

91

$\mathrm{R}^{2}=\mathrm{H}, \mathrm{CH}_{3}$

XXVIII

(10 mol\%)

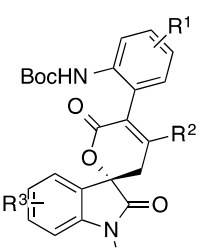

$P G$

93

$84-99 \%$ Y (87- $99 \%$ ee)

$\wedge$<smiles>CC(C)O[Mg]</smiles><smiles></smiles>

$\overrightarrow{\text { Si face addition }}$

92

$\mathrm{R}^{2}=\mathrm{H}, \mathrm{CH}_{3}$ $s$-cis enolate

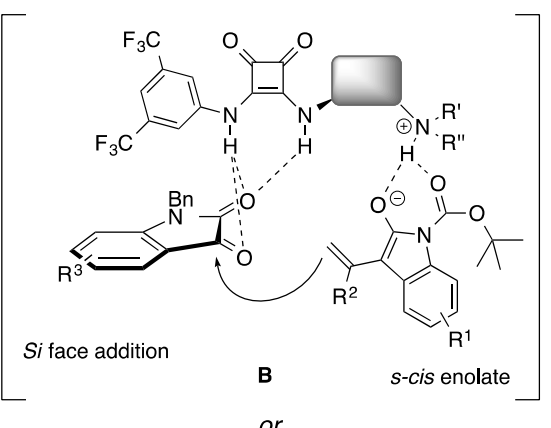

or

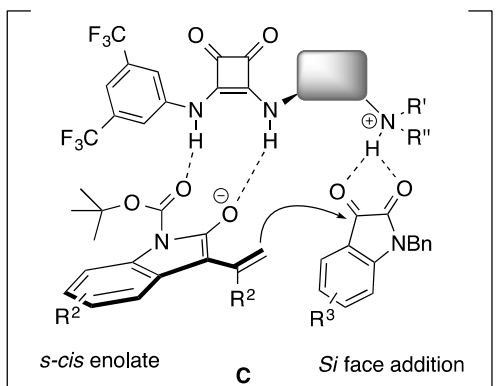

C Si face addition

$=$ Chiral Scaffold

Scheme 27. Asymmetric aldol/lactonization sequence for the synthesis of spirooxindole dihydropyranones 93. A is the hypothesized intermediate on the reaction pathway, while $\mathbf{B}$ and $\mathbf{C}$ are proposed alternative models for explaining the dual activation of nucleophile and electrophile.

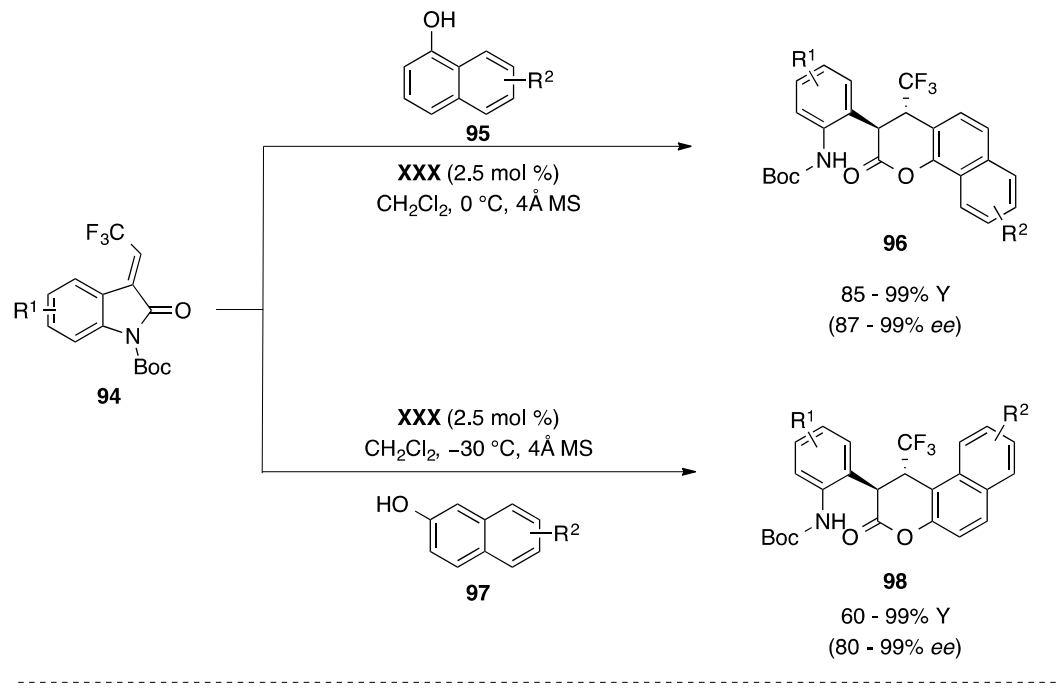

Proposed reaction pathway

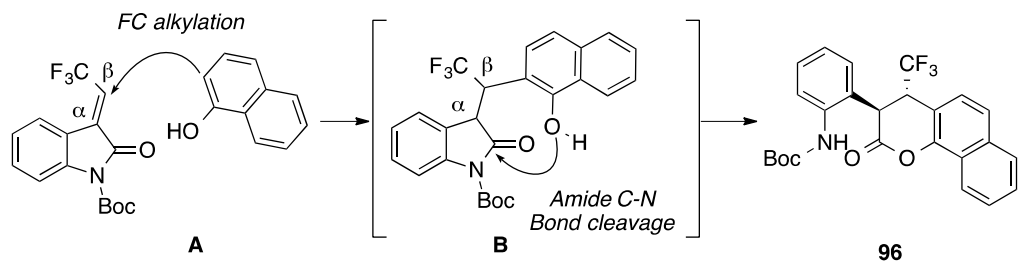

$$
\begin{gathered}
96 \\
\left(R^{1}=R^{2}=H\right)
\end{gathered}
$$

Scheme 28. Friedel-Crafts/lactonization domino reaction developed by Zhao and co-workers and suggested reaction pathway. 
Although the lactonization occurred to disadvantage of the chemically inert oxindole unit, the overall process proceeded under very mild conditions (i.e., $\mathrm{CH}_{2} \mathrm{Cl}_{2}$ at low temperature) and affording attractive outcomes in term of yields (60-99\%) and stereoselectivity (>20:1 d.r., $80-98 \% e e$ ) when both $\alpha$ - and $\beta$-naphthols (95 and 97) were alternatively used as nucleophiles (Scheme 28).

Cinchona-derived squaramides have shown their potential as organocatalysts even when they are involved in more complex domino reactions. An interesting example was reported by Lu and co-workers in 2015 who initially optimize a simple Diels-Alder/aromatization sequence and then, by adding an excess of oxindole moiety, observed a Diels-Alder/Michael/aromatization domino process (Scheme 29) [65]. The reactions always proceeded smoothly under very mild conditions (i.e., $\mathrm{CH}_{2} \mathrm{Cl}_{2}$ at room temperature) and with quite low catalyst loading (10 mol \% of XXVI) providing the expected carbazolespirooxindole derivatives 101 in moderate-to-good yields (48-90\%) and interesting stereoinduction (from 4:1 to >20:1 d.r., 60-99\% ee). Unfortunately, for the product 102 with six contiguous stereocenters furnished by the triple domino reaction, it was not possible to define the stereochemical outcome mainly due to the arduous separation of the complex diastereomeric mixture.
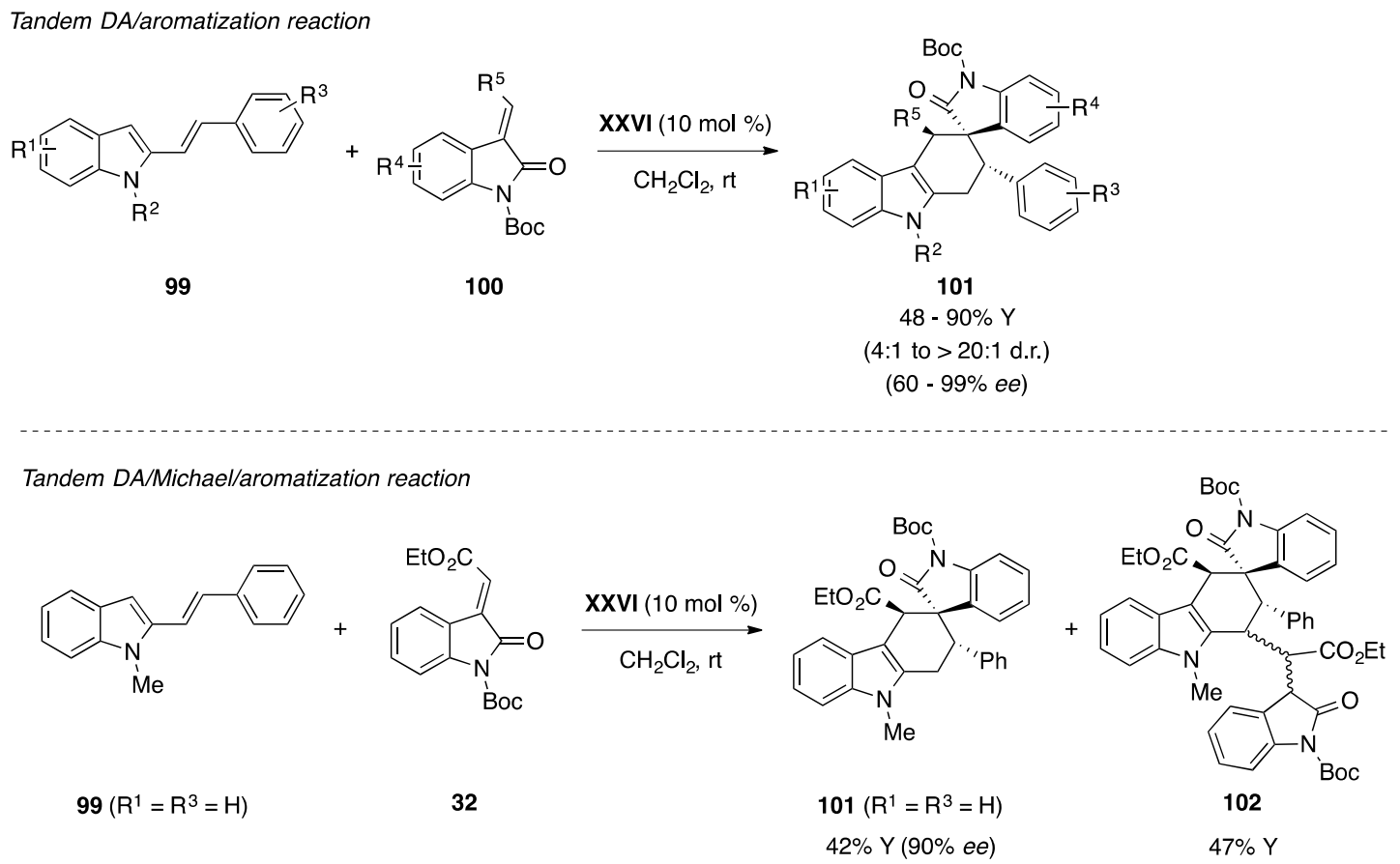

Scheme 29. Diels-Alder (DA)/aromatization sequence and triple DA/Michael/aromatization domino process.

\section{Miscellaneous}

The impressive reactivity of oxindole derivatives has found several notable applications even when phosphoric acids, a less explored class of catalophores are employed as Brønsted acid organocatalyst. Among the very few reported examples, Shi and co-workers rationally designed a chiral phosphoric acid (CPA)-catalyzed Michael addition/intramolecular Friedel-Crafts cascade reaction toward the construction of cyclopenta[b]indole and spirooxindole frameworks [66]. Specifically, the authors supposed that, in order to promote the initial vinylogous Michael addition, the CPA XXXI could simultaneously activate throughout a complex H-bond network both the 7-vinylindoles 103 as nucleophile and the electrophilic vinyliminium C, which should be generated in situ form the 3-indolylmethanols 104. Subsequently the transient adduct $\mathbf{B}$ should undergo, always assisted by the dual H-bond activation of CPA XXXI, the intramolecular Friedel-Crafts reaction to 
restore the initial aromatic indolic structure and furnish the expected complex spirooxindole systems 105 (Scheme 30).<smiles>[R]C=Cc1cccc2c([R7])c[nH]c12</smiles>

103<smiles>O=C1N([Tl])c2ccccc2C1(O)c1c[nH]c2ccccc12</smiles>

104

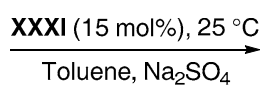

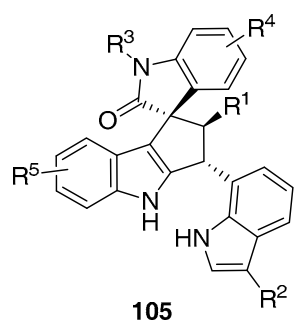

$41-78 \%$ Y

$(>95: 5 \mathrm{dr} ; 94->99 \%$ ee)
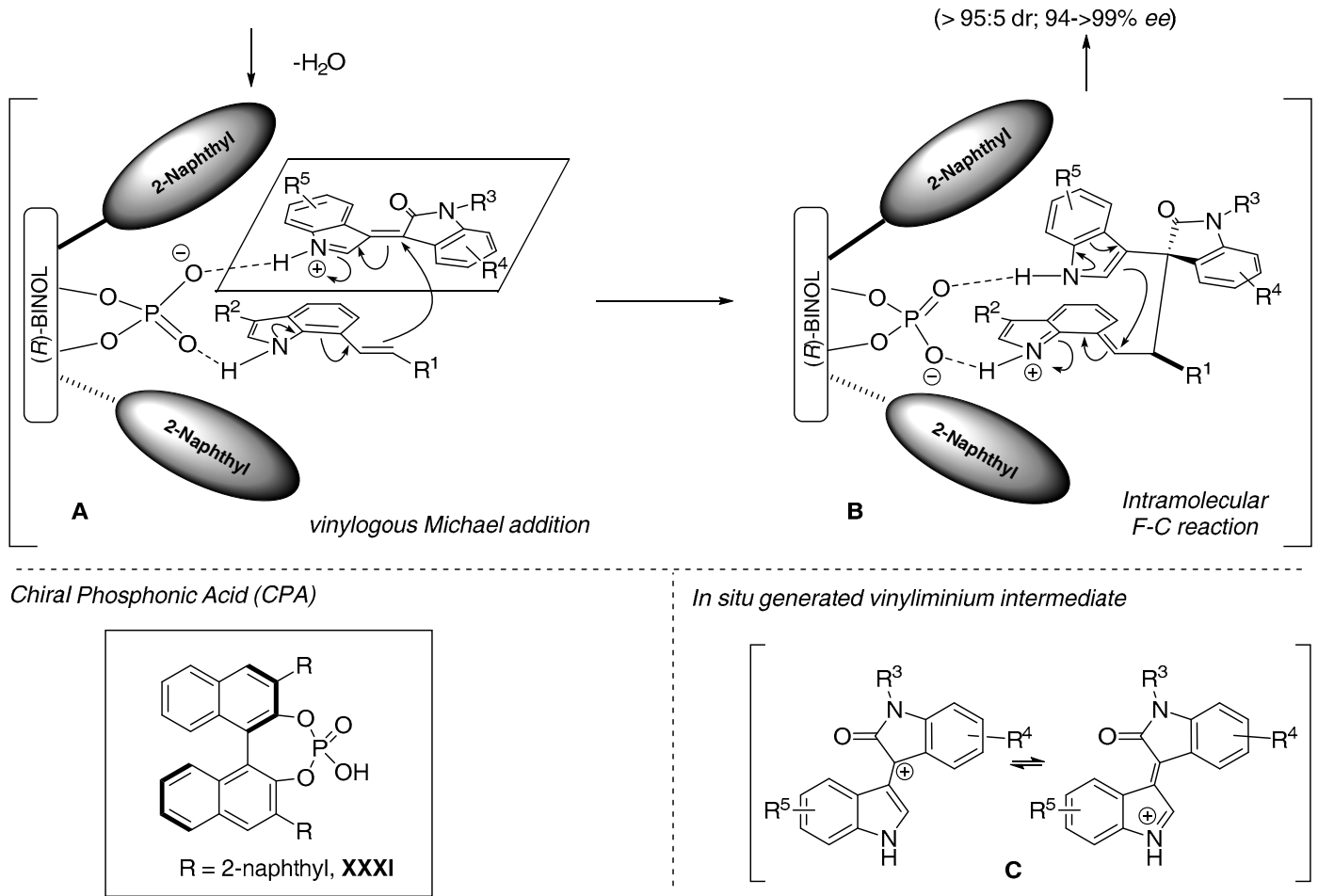

B

In situ generated vinyliminium intermediate

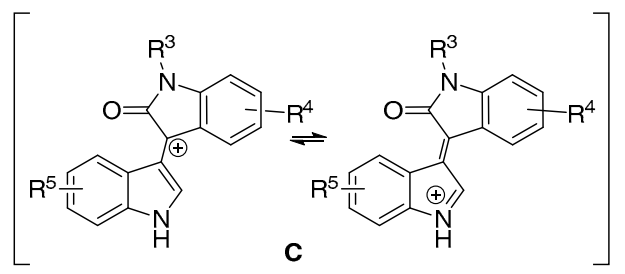

Scheme 30. (CPA)-catalyzed Michael addition/intramolecular Friedel-Crafts cascade reaction toward the construction of cyclopenta[b]indole and spirooxindole scaffold. A-C are postulated intermediates on the mechanism of the reaction.

The authors demonstrated their hypothesis successfully performing the diastereo- and enantioselective synthesis of several cyclopenta[b]indole derivatives $\mathbf{1 0 5}$ starting from a series of 7-vinylindoles 103 and various isatin-derived 3-indolylmethanols 104. Comparing the obtained results, it was clear that the stereoinduction was essentially not affected by the different nature of the substituents on both acceptor and donor partners. Conversely, they dramatically influenced the overall reactivity. Particularly, electron-donating groups on C7-functionalised indoles gave much higher yields than the electron-withdrawing groups, while it was less explainable the electronic effect due to substituents on the isatin units.

\section{Conclusions}

As shown by the examples depicted here, when newly developed organocatalysts meet oxindole derivatives, one of the most widespread frameworks in Nature, it is possible to achieve impressive levels of molecular complexity featured by the formation of multiple stereogenic centers upon multiple cascade reactions. Such outstanding results and all the future predictable improvements not only are going to constantly provide easier and easier access to a broad spectrum of highly valuable complex 
compounds, but also to furnish a better understanding of Nature's modes of action and ultimately even reach an almost similar degree of perfection.

Acknowledgments: Tecla Gasperi and Martina Miceli gratefully acknowledge Dipartimento di Scienze and Sezione di Nanoscienze e Nanotecnologie (Università si Roma Tre, Roma, Italy) for the financial support. Jean-Marc Campagne and Renata Marcia de Figueiredo are grateful to the support from ENSCM and CNRS.

Author Contributions: Tecla Gasperi, Martina Miceli, Jean-Marc Campagne, and Renata Marcia de Figueiredo conceived, wrote and revised the review.

Conflicts of Interest: The authors declare no conflict of interest.

\section{References}

1. Wang, Y.; Lu, H.; Xu, P.F. Asymmetric catalytic cascade reactions for constructing diverse scaffolds and complex molecules. Acc. Chem. Res. 2015, 48, 1832-1844. [CrossRef] [PubMed]

2. Cao, Z.-Y.; Zhou, J. Catalytic asymmetric synthesis of polysubstituted spirocyclopropyl oxindoles: Organocatalysis versus transition metal catalysis. Org. Chem. Front. 2015, 2, 849-858. [CrossRef]

3. Trost, B.; Brennan, M. Asymmetric syntheses of oxindole and indole spirocyclic alkaloid natural products. Synthesis 2009, 18, 3003-3025. [CrossRef]

4. Antonchick, A.P.; Gerding-Reimers, C.; Catarinella, M.; Schurmann, M.; Preut, H.; Ziegler, S.; Rauh, D.; Waldmann, H. Highly enantioselective synthesis and cellular evaluation of spirooxindoles inspired by natural products. Nat. Chem. 2010, 2, 735-740. [CrossRef] [PubMed]

5. Yu, B.; Xing, H.; Yu, D.Q.; Liu, H.M. Catalytic asymmetric synthesis of biologically important 3-hydroxyoxindoles: An update. Beilstein J. Org. Chem. 2016, 12, 1000-1039. [CrossRef] [PubMed]

6. Kaur, J.; Chimni, S.S.; Mahajan, S.; Kumar, A. Stereoselective synthesis of 3-amino-2-oxindoles from isatin imines: New scaffolds for bioactivity evaluation. RSC Adv. 2015, 5, 52481-52496. [CrossRef]

7. Tietze, L.F.; Brasche, G.; Gericke, K.M. Domino Reaction in Organic Synthesis; Wiley-VCH: Weinheim, Germany, 2006.

8. Volla, C.M.; Atodiresei, I.; Rueping, M. Catalytic c-c bond-forming multi-component cascade or domino reactions: Pushing the boundaries of complexity in asymmetric organocatalysis. Chem. Rev. 2014, 114, 2390-2431. [CrossRef] [PubMed]

9. Grondal, C.; Jeanty, M.; Enders, D. Organocatalytic cascade reactions as a new tool in total synthesis. Nat. Chem. 2010, 2, 167-178. [CrossRef] [PubMed]

10. Tian, L.; Luo, Y.-C.; Hu, X.-Q.; Xu, P.-F. Recent developments in the synthesis of chiral compounds with quaternary centers by organocatalytic cascade reactions. Asian J. Org. Chem. 2016, 5, 580-607. [CrossRef]

11. Gasperi, T.; Vetica, F.; de Figueiredo, R.; Orsini, M.; Tofani, D. Recent advances in organocatalytic cascade reactions toward the formation of quaternary stereocenters. Synthesis 2015, 47, 2139-2184. [CrossRef]

12. Hong, L.; Wang, R. Recent advances in asymmetric organocatalytic construction of 3,3'-spirocyclic oxindoles. Adv. Synth. Catal. 2013, 355, 1023-1052. [CrossRef]

13. Bencivenni, G.; Wu, L.Y.; Mazzanti, A.; Giannichi, B.; Pesciaioli, F.; Song, M.P.; Bartoli, G.; Melchiorre, P. Targeting structural and stereochemical complexity by organocascade catalysis: Construction of spirocyclic oxindoles having multiple stereocenters. Angew. Chem. Int. Ed. 2009, 48, 7200-7203. [CrossRef] [PubMed]

14. Hof, K.; Lippeert, K.M.; Schreiner, P.R. Science of Synthesis: Asymmetric Organocatalysis 2; Georg Thieme Verlag KG: Stuttgart, Germany, 2012.

15. Knowles, R.R.; Jacobsen, E.N. Attractive non-covalent interactions in asymmetric catalysis: Links between enzymes and small molecule catalysts. Proc. Natl. Acad. Sci. USA 2010, 107, 20678-20685. [CrossRef] [PubMed]

16. Etzenbach-Effers, K.; Berkessel, A. Non-covalent organocatalysis based on hydrogen bonding: Elucidation of reaction paths by computational methods. In Asymmetric Organocatalysis; List, B., Ed.; Springer: Berlin, Germany, 2009; Volume 291, pp. 1-27.

17. De Figueiredo, R.M.; Mazziotta, A.; de Sant'Ana, D.P.; Palumbo, C.; Gasperi, T. Active methylene compounds in asymmetric organocatalytic synthesis of natural products and pharmaceutical scaffolds. Curr. Org. Chem. 2012, 16, 2231-2289. [CrossRef]

18. Song, C.E. Cinchona Alkaloids in Synthesis and Catalysis, Ligands, Immobilization and Organocatalysis; WILEY-VCH Verlag GmbH \& Co, KGaA: Weinheim, Germany, 2009. 
19. Bai, M.; Cui, B.-D.; Zuo, J.; Zhao, J.-Q.; You, Y.; Chen, Y.-Z.; Xu, X.-Y.; Zhang, X.-M.; Yuan, W.-C. Quinine-catalyzed asymmetric domino Mannich-cyclization reactions of 3-isothiocyanato oxindoles with imines for the synthesis of spirocyclic oxindoles. Tetrahedron 2015, 71, 949-955. [CrossRef]

20. Huang, J.R.; Sohail, M.; Taniguchi, T.; Monde, K.; Tanaka, F. Formal [4 + 1] cycloaddition and enantioselective Michael-Henry cascade reactions to synthesize spiro[4,5]decanes and spirooxindole polycycles. Angew. Chem. Int. Ed. Eng. 2017, 56, 5853-5857. [CrossRef] [PubMed]

21. Li, T.-Z.; Xie, J.; Jiang, Y.; Sha, F.; Wu, X.-Y. Enantioselective vinylogous Michael/cyclization cascade reaction of acyclic $\beta, \gamma$-unsaturated amides with isatylidene malononitriles: Asymmetric construction of spirocyclic oxindoles. Adv. Synth. Catal. 2015, 357, 3507-3511. [CrossRef]

22. Xie, J.; Xing, X.Y.; Sha, F.; Wu, Z.Y.; Wu, X.Y. Enantioselective synthesis of spiro[indoline-3,4'-pyrano[2,3-c]pyrazole] derivatives via an organocatalytic asymmetric michael/cyclization cascade reaction. Org. Biomol. Chem. 2016, 14, 8346-8355. [CrossRef] [PubMed]

23. Zhao, K.; Zhi, Y.; Li, X.; Puttreddy, R.; Rissanen, K.; Enders, D. Asymmetric synthesis of 3,3'-pyrrolidinyl-dispirooxindoles via a one-pot organocatalytic Mannich/deprotection/aza-Michael sequence. Chem. Commun. 2016, 52, 2249-2252. [CrossRef] [PubMed]

24. Connon, S.J. Organocatalysis mediated by (thio)urea derivatives. Chem. Eur. J. 2006, 12, 5418-5427. [CrossRef] [PubMed]

25. Takemoto, Y. Development of chiral thiourea catalysts and its application to asymmetric catalytic reactions. Chem. Pharm. Bull. 2010, 58, 593-601. [CrossRef] [PubMed]

26. Fang, X.; Wang, C.J. Recent advances in asymmetric organocatalysis mediated by bifunctional amine-thioureas bearing multiple hydrogen-bonding donors. Chem. Commun. 2015, 51, 1185-1197. [CrossRef] [PubMed]

27. Sun, Y.-L.; Wei, Y.; Shi, M. Applications of chiral thiourea-amine/phosphine organocatalysts in catalytic asymmetric reactions. Chem CatChem 2017, 9, 718-727. [CrossRef]

28. Reddy Gajulapalli, V.P.; Vinayagam, P.; Kesavan, V. Enantioselective assembly of functionalized carbocyclic spirooxindoles using anl-proline derived thiourea organocatalyst. RSC Adv. 2015, 5, 7370-7379. [CrossRef]

29. Auria-Luna, F.; Marques-Lopez, E.; Mohammadi, S.; Heiran, R.; Herrera, R.P. New organocatalytic asymmetric synthesis of highly substituted chiral 2-oxospiro-[indole-3, $4^{\prime}-\left(1^{\prime}, 4^{\prime}\right.$-dihydropyridine)] derivatives. Molecules 2015, 20, 15807-15826. [CrossRef] [PubMed]

30. Okino, T.; Hoashi, Y.; Takemoto, Y. Enantioselective michael reaction of malonates to nitroolefins catalyzed by bifunctional organocatalysts. J. Am. Chem. Soc. 2003, 125, 12672-12673. [CrossRef] [PubMed]

31. Xie, J.; Xing, W.-L.; Sha, F.; Wu, X.-Y. Enantioselective cascade reaction of $\alpha$-cyano ketones and isatylidene malononitriles: Asymmetric construction of spiro[4h-pyran-oxindoles]. Eur. J. Org. Chem. 2016, 2016, 3983-3992. [CrossRef]

32. Cui, L.Y.; Wang, Y.M.; Zhou, Z.H. Enantioselective construction of novel chiral spirooxindoles incorporating a thiazole nucleus. RSC Adv. 2016, 6, 64474-64481. [CrossRef]

33. Pratap Reddy Gajulapalli, V.; Lokesh, K.; Vishwanath, M.; Kesavan, V. Organocatalytic construction of spirooxindole naphthoquinones through Michael/hemiketalization using L-proline derived bifunctional thiourea. RSC Adv. 2016, 6, 12180-12184. [CrossRef]

34. Yin, S.-J.; Zhang, S.-Y.; Zhang, J.-Q.; Sun, B.-B.; Fan, W.-T.; Wu, B.; Wang, X.-W. Organocatalytic tandem enantioselective Michael-cyclization of isatin-derived $\beta, \gamma$-unsaturated $\alpha$-ketoesters with 3-hydroxy-4h-chromen-4-one or 2-hydroxy-1,4-naphthoquinone derivatives. RSC Adv. 2016, 6, 84248-84254. [CrossRef]

35. Zhao, K.; Zhi, Y.; Shu, T.; Valkonen, A.; Rissanen, K.; Enders, D. Organocatalytic domino oxa-Michael/1,6-addition reactions: Asymmetric synthesis of chromans bearing oxindole scaffolds. Angew. Chem. Int. Ed. 2016, 55, 12104-12108. [CrossRef] [PubMed]

36. Chowdhury, R.; Kumar, M.; Ghosh, S.K. Organocatalyzed enantioselective Michael addition/cyclization cascade reaction of 3-isothiocyanato oxindoles with arylidene malonates. Org. Biomol. Chem. 2016, 14, 11250-11260. [CrossRef] [PubMed]

37. Wu, C.; Jing, L.; Qin, D.; Yin, M.; He, Q. Organocatalytic asymmetric synthesis of trans-configured trispirooxindoles through a cascade Michael-cyclization reaction. Tetrahedron Lett. 2016, 57, 2857-2860. [CrossRef] 
38. Zhao, J.Q.; Zhou, M.Q.; Wu, Z.J.; Wang, Z.H.; Yue, D.F.; Xu, X.Y.; Zhang, X.M.; Yuan, W.C. Asymmetric Michael/cyclization cascade reaction of 3-isothiocyanato oxindoles and 3-nitroindoles with amino-thiocarbamate catalysts: Enantioselective synthesis of polycyclic spirooxindoles. Org. Lett. 2015, 17, 2238-2241. [CrossRef] [PubMed]

39. Yi, W.-B.; Zhang, Z.; Huang, X.; Tanner, A.; Cai, C.; Zhang, W. One-pot fluorination and asymmetric Michael addition promoted by recyclable fluorous organocatalysts. RSC Adv. 2013, 3, 18267-18270. [CrossRef]

40. Huang, X.; Pham, K.; Yi, W.; Zhang, X.; Clamens, C.; Hyatt, J.H.; Jasinsk, J.P.; Tayvah, U.; Zhang, W. Recyclable organocatalyst-promoted one-pot asymmetric synthesis of spirooxindoles bearing multiple stereogenic centers. Adv. Synth. Catal. 2015, 357, 3820-3824. [CrossRef]

41. Huang, X.; Liu, M.; Pham, K.; Zhang, X.; Yi, W.B.; Jasinski, J.P.; Zhang, W. Organocatalytic one-pot asymmetric synthesis of thiolated spiro- $\gamma$-lactam oxindoles bearing three stereocenters. J. Org. Chem. 2016, 81, 5362-5369. [CrossRef] [PubMed]

42. Zhang, W.; Curran, D.P. Synthetic applications of fluorous solid-phase extraction (f-spe). Tetrahedron 2006, 62, 11837-11865. [CrossRef] [PubMed]

43. Enders, D.; Zhi, Y.; Zhao, K.; von Essen, C.; Rissanen, K. Thiourea-catalyzed domino Michael-Mannich [3 + 2] cycloadditions: A strategy for the asymmetric synthesis of 3,3'-pyrrolidinyl-dispirooxindoles. Synlett 2017. [CrossRef]

44. Monari, M.; Montroni, E.; Nitti, A.; Lombardo, M.; Trombini, C.; Quintavalla, A. Highly stereoselective [4 + 2] and [3 + 2] spiroannulations of 2-(2-oxoindolin-3-ylidene)acetic esters catalyzed by bifunctional thioureas. Chem. Eur. J. 2015, 21, 11038-11049. [CrossRef] [PubMed]

45. Cerisoli, L.; Lombardo, M.; Trombini, C.; Quintavalla, A. The first enantioselective organocatalytic synthesis of 3-spiro- $\alpha$-alkylidene-gamma-butyrolactone oxindoles. Chem. Eur. J. 2016, 22, 3865-3872. [CrossRef] [PubMed]

46. Quintavalla, A.; Lanza, F.; Montroni, E.; Lombardo, M.; Trombini, C. Organocatalytic conjugate addition of nitroalkanes to 3-ylidene oxindoles: A stereocontrolled diversity oriented route to oxindole derivatives. J. Org. Chem. 2013, 78, 12049-12064. [CrossRef] [PubMed]

47. Muthusamy, S.; Prakash, M.; Ramakrishnan, C.; Gromiha, M.M.; Kesavan, V. Organocatalytic enantioselective assembly of spirooxindole-naphthopyrans through tandem Friedel-Crafts type/hemiketalization. ChemCatChem 2016, 8, 1708-1712. [CrossRef]

48. Vishwanath, M.; Vinayagam, P.; Gajulapalli, V.P.R.; Kesavan, V. Asymmetric organocatalytic assembly of oxindoles fused with spiro-3,4-dihydropyrans with three contiguous stereocenters consisting of vicinal quaternary centers. Asian J. Org. Chem. 2016, 5, 613-616. [CrossRef]

49. Du, D.; Jiang, Y.; Xu, Q.; Tang, X.-Y.; Shi, M. Enantioselective [3 + 2] cyclization of 3-isothiocyanato oxindoles with trifluoromethylated 2-butenedioic acid diesters. Chem CatChem 2015, 7, 1366-1371. [CrossRef]

50. Cook, A.G.; Voges, A.B.; Kammarath, A.E. Aminal-catalyzed isomerization of and addition to dimethyl maleate. Tetrahedron Lett. 2001, 42, 7349-7352. [CrossRef]

51. Malerich, J.P.; Hagihara, K.; Rawal, V.H. Chiral squaramide derivatives are excellent hydrogen bond donor catalyst. J. Am. Chem. Soc. 2008, 130, 14416-14417. [CrossRef] [PubMed]

52. Zhao, B.-L.; Du, D.-M. Organocatalytic enantioselective cascade aza-Michael/Michael addition sequence for asymmetric synthesis of chiral spiro[pyrrolidine-3,3'-oxindole]s. Asian J. Org. Chem. 2015, 4, 1120-1126. [CrossRef]

53. Zhao, B.L.; Du, D.M. Organocatalytic cascade Michael/Michael reaction for the asymmetric synthesis of spirooxindoles containing five contiguous stereocenters. Chem. Commun. 2016, 52, 6162-6165. [CrossRef] [PubMed]

54. Zhao, B.-L.; Du, D.-M. Squaramide-catalyzed enantioselective cascade approach to bispirooxindoles with multiple stereocenters. Adv. Synth. Catal. 2016, 358, 3992-3998. [CrossRef]

55. Chen, L.; Wu, Z.J.; Zhang, M.L.; Yue, D.F.; Zhang, X.M.; Xu, X.Y.; Yuan, W.C. Organocatalytic asymmetric Michael/cyclization cascade reactions of 3-hydroxyoxindoles/3-aminooxindoles with $\alpha, \beta$-unsaturated acyl phosphonates for the construction of spirocyclic oxindole- $\gamma$-lactones/lactams. J. Org. Chem. 2015, 80, 12668-12675. [CrossRef] [PubMed]

56. Ming, S.; Zhao, B.L.; Du, D.M. Chiral squaramide-catalysed enantioselective Michael/cyclization cascade reaction of 3-hydroxyoxindoles with $\alpha, \beta$-unsaturated $N$-acylated succinimides. Org. Biomol. Chem. 2017, 15, 6205-6213. [CrossRef] [PubMed] 
57. Lin, Y.; Liu, L.; Du, D.-M. Squaramide-catalyzed asymmetric Michael/cyclization cascade reaction of 3-isothiocyanato oxindoles with chalcones for synthesis of pyrrolidinyl spirooxindoles. Org. Chem. Front. 2017, 4, 1229-1238. [CrossRef]

58. Liu, L.; Zhao, B.-L.; Du, D.-M. Organocatalytic asymmetric Michael/cyclization cascade reaction of 3-isothiocyanato oxindoles with maleimides for the efficient construction of pyrrolidonyl spirooxindoles. Eur. J. Org. Chem. 2016, 2016, 4711-4718. [CrossRef]

59. Lou, Q.; Ding, Y.; Xu, D.; Liu, G.; Zhao, J. Organocatalytic enantioselective synthesis of dihydropyranoindole derivatives bearing trifluoromethylated all-carbon-substituted stereocenters. Adv. Synth. Catal. 2017, 359, 2557-2563. [CrossRef]

60. Curti, C.; Rassu, G.; Zambrano, V.; Pinna, L.; Pelosi, G.; Sartori, A.; Battistini, L.; Zanardi, F.; Casiraghi, G. Bifunctional cinchona alkaloid/thiourea catalyzes direct and enantioselective vinylogous Michael addition of 3-alkylidene oxindoles to nitroolefins. Angew. Chem. Int. Ed. Engl. 2012, 51, 6200-6204. [CrossRef] [PubMed]

61. Rassu, G.; Zambrano, V.; Pinna, L.; Curti, C.; Battistini, L.; Sartori, A.; Pelosi, G.; Zanardi, F.; Casiraghi, G. Direct regio-, diastereo-, and enantioselective vinylogous Michael addition of prochiral 3-alkylideneoxindoles to nitroolefins. Adv. Synth. Catal. 2013, 355, 1881-1886. [CrossRef]

62. Rassu, G.; Zambrano, V.; Tanca, R.; Sartori, A.; Battistini, L.; Zanardi, F.; Curti, C.; Casiraghi, G. 3-alkenyl-2-silyloxyindoles: An enabling, yet understated progeny of vinylogous carbon nucleophiles. Eur. J. Org. Chem. 2012, 2012, 466-470. [CrossRef]

63. Han, J.L.; Chang, C.H. An asymmetric assembly of spirooxindole dihydropyranones through a direct enantioselective organocatalytic vinylogous aldol-cyclization cascade reaction of 3-alkylidene oxindoles with isatins. Chem. Commun. 2016, 52, 2322-2325. [CrossRef] [PubMed]

64. Zhao, Y.L.; Lou, Q.X.; Wang, L.S.; Hu, W.H.; Zhao, J.L. Organocatalytic Friedel-Crafts alkylation/lactonization reaction of naphthols with 3-trifluoroethylidene oxindoles: The asymmetric synthesis of dihydrocoumarins. Angew. Chem. Int. Ed. Eng. 2017, 56, 338-342. [CrossRef] [PubMed]

65. Huang, L.-J.; Weng, J.; Wang, S.; Lu, G. Organocatalytic diels-alder reaction of 2-vinylindoles with methyleneindolinones: An efficient approach to functionalized carbazolespirooxindoles. Adv. Synth. Catal. 2015, 357, 993-1003. [CrossRef]

66. Shi, F.; Zhang, H.H.; Sun, X.X.; Liang, J.; Fan, T.; Tu, S.J. Organocatalytic asymmetric cascade reactions of 7-vinylindoles: Ddiastereo- and enantioselective synthesis of C7-functionalized indoles. Chem. Eur. J. 2015, 21, 3465-3471. [CrossRef] [PubMed] 